\title{
Quantum Fluctuations and Large Deviation Principle for Microscopic Currents of Free Fermions in Disordered Media
}

\author{
J.-B. Bru W. de Siqueira Pedra A. Ratsimanetrimanana \\ May 27, 2021
}

\begin{abstract}
We contribute an extension of large-deviation results obtained in [N.J.B. Aza, J.-B. Bru, W. de Siqueira Pedra, A. Ratsimanetrimanana, J. Math. Pures Appl. 125 (2019) 209] on conductivity theory at atomic scale of free lattice fermions in disordered media. Disorder is modeled by (i) a random external potential, like in the celebrated Anderson model, and (ii) a nearest-neighbor hopping term with random complex-valued amplitudes. In accordance with experimental observations, via the large deviation formalism, our previous paper showed in this case that quantum uncertainty of microscopic electric current densities around their (classical) macroscopic value is suppressed, exponentially fast with respect to the volume of the region of the lattice where an external electric field is applied. Here, the quantum fluctuations of linear response currents are shown to exist in the thermodynamic limit and we mathematically prove that they are related to the rate function of the large deviation principle associated with current densities. We also demonstrate that, in general, they do not vanish (in the thermodynamic limit) and the quantum uncertainty around the macroscopic current density disappears exponentially fast with an exponential rate proportional to the squared deviation of the current from its macroscopic value and the inverse current fluctuation, with respect to growing space (volume) scales.
\end{abstract}

Keywords: Quantum fluctuations, large deviations, Fermionic charge transport, disordered media.

\section{Contents}

\begin{tabular}{llr}
\hline 1 & Introduction & 2
\end{tabular}

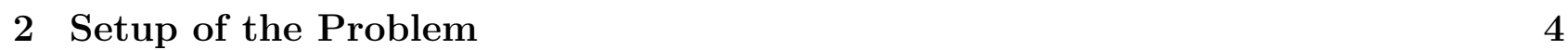

2.1 Random Tight-Binding Model . . . . . . . . . . . . . . . . . . . . . . . . . . . . . . . . . .

$2.2 \quad C^{*}$-Algebraic Setting . . . . . . . . . . . . . . . . . . . . . . . 5

2.3 Linear Response Current Density . . . . . . . . . . . . . . . . . . . . . . 6

$2.4 \quad$ Large Deviations for Microscopic Current Densities . . . . . . . . . . . . . . . 7

\begin{tabular}{llr}
\hline 3 & Main Results & $\mathbf{8}$ \\
\hline
\end{tabular}

$3.1 \quad$ Quantum Fluctuations of Linear Response Currents and Rate Function . . . . . 8

3.2 Non-Vanishing Quantum Fluctuations of Linear Response Currents . . . . . . . 10 
4.1 Quasi-Free Fermions in Subregions of the Lattice . . . . . . . . . . . . . . . . . 11

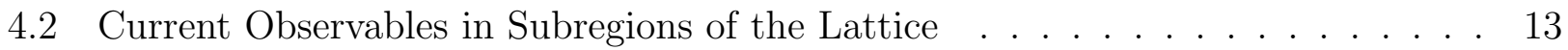

4.3 Differentiability Class of Generating Functions . . . . . . . . . . . . . . . . . . 14

4.4 Non-Vanishing Second Derivative of Generating Functions at the Origin . . . . . 19

\section{Introduction}

Surprisingly [2], in 2012, experimental measurements [3] of electric resistance of nanowires in Si doped with phosphorus atoms demonstrate that the macroscopic laws for charge transport are already accurate at length scales larger than a few nanometers, even at very low temperature $(4.2 \mathrm{~K})$. As a consequence, microscopic (quantum) effects on charge transport can very rapidly disappear with respect to growing space scales. Understanding the breakdown of the classical (macroscopic) conductivity theory at microscopic scales is an important technological issue, because of the growing need for smaller electronic components.

From a mathematical perspective, the convergence of the expectations of microscopic current densities with respect to growing space scales is proven in [5, 6], but no information about the suppression of quantum uncertainty was obtained in the macroscopic limit. In [1], in accordance with experimental observations, it is proven, for non-interacting lattice fermions with disorder, that quantum uncertainty of microscopic electric current densities around their (classical) macroscopic value is suppressed, exponentially fast with respect to the volume of the region of the lattice where an external electric field is applied. This is proven in [1] via the large deviation formalism [7, 8], which has been adopted in quantum statistical mechanics since the eighties [9, Section 7]. Given a fixed electromagnetic field $\mathcal{E}$, we derive in particular in [1] the (good) rate function $\mathrm{I}^{(\mathcal{E})}$ associated with microscopic (linear response) current densities $\mathrm{1}^{1}$ $x_{L}^{(\mathcal{E})} \in \mathbb{R}, L \in \mathbb{R}_{0}^{+}$, meaning in this case that, in a cubic box of volume $L^{d}$ ( $d$-dimmensional lattice), for any $a, b \in \mathbb{R}$,

$$
\operatorname{Prob}\left[x_{L}^{(\mathcal{E})} \in[a, b]\right] \sim \mathrm{e}^{-L^{d} \inf _{x \in[a, b]} \mathrm{I}^{(\mathcal{E})}(x)}, \quad \text { as } L \rightarrow \infty,
$$

with $\mathrm{I}^{(\mathcal{E})} \geq 0$ and $\mathrm{I}^{(\mathcal{E})}(x)=0$ iff $x$ is the macroscopic (linear response) current density, $x^{(\mathcal{E})}$.

In this paper, we complement these studies by rigorously showing two new properties of charge transport of quasi-free fermions in disordered media:

(a) The quantum fluctuations of linear response currents exist in the thermodynamic limit and are meanwhile explicitly related to the rate function $\mathrm{I}^{(\mathcal{E})}$, as expected.

(b) In general, the quantum fluctuations of currents do not vanish in the thermodynamic limit and the quantum uncertainty around the macroscopic current density disappears exponentially fast with an exponential rate proportional to $\left(x-x^{(\mathcal{E})}\right)^{2}$ and the inverse current fluctuation, with respect to growing space (volume) scales.

(a)-(b) refer to Theorems 3.1 and 3.3, which are the main results of this paper.

Our results show that the experimental measure of the rate function $\mathrm{I}^{(\mathcal{E})}$ (see $(1)$ ) leads to an experimental estimate on the corresponding quantum fluctuations. Conversely, an experimental estimate on these quantum fluctuations gives the behavior of the corresponding rate function $\mathrm{I}^{(\mathcal{E})}$ around the macroscopic current density $x^{(\mathcal{E})}$. This fact is certainly not restricted to fermionic currents.

\footnotetext{
${ }^{1}$ In some direction of $\mathbb{R}^{d}$.
} 
Note that the existence of quantum fluctuations and associated mathematical structures has been extensively studied for quantum many-body systems. This refers for instance to the construction of so-called algebra of normal fluctuations for transport phenomena, which are related to quantum central limit theorems. See, e.g., [5, 10, 11, 12, 13, 14, 15, 16] as well as [17, Chapter 6] and references therein. The explicit relation (a) we derive between quantum fluctuations and the large deviation formalism in quantum statistical mechanics [9, Section 7] is, however, a new general observation on quantum many-body systems.

We use the mathematical framework of [1, 6, 18, to study fermions on the lattice. For simplicity we take a cubic lattice $\mathbb{Z}^{d}$, even if other types of lattices can be considered with very similar methods. Disorder within the conductive material, due to impurities, crystal lattice defects, etc., is modeled by (i) a random external potential, like in the celebrated Anderson model, and (ii) a nearest-neighbor hopping term with random complex-valued amplitudes. In particular, random (electromagnetic) vector potentials can also be implemented. The celebrated tight-binding Anderson model is one particular example of the general case considered here.

In order to prove Property (a), i.e., Theorem 3.1, we use the large deviation formalism and follow the argument lines of [1, Section 4] to show [1, Theorem 3.1] via the Akcoglu-Krengel ergodic theorem [1, Theorem 4.17], for one has to control the thermodynamical limit of (finitevolume) generating functions that are random. We perform in particular the same box decomposition of these random functions, which can be justified with the help of the Bogoliubov-type inequality [1, Lemma 4.2] and the "locality" (or space decay) of both the quasi-free dynamics and space correlations of KMS states, which is a consequence of Combes-Thomas estimates [1, Appendix A]. See [1, Section 4.3]. In this paper we only give the new arguments that are necessary to prove Property (a), like the existence of the thermodynamic limit of quantum fluctuations of currents and the continuity of the second derivative of the generating function. In particular, like in the proof of [1, Corollary 4.20], we use the (Arzelà-) Ascoli theorem [19, Theorem A5], which requires uniform bounds on the third-order derivatives of finite-volume generating functions. This proof is much more computational than the one of [1, Proposition 4.9], which only control the first and second derivatives of the same function. Note that derivatives of the logarithm of the expectations of an exponential, like the generating function we consider here, are generally related to so-called "truncated" or "connected" correlations. We demonstrate that it is the case for the third-order derivative we refer to above, allowing the reader to follow the computation of that derivative in a systematic way. Considering the third-order case, the algorithm to compute the derivatives of the generating functions at any order becomes apparent, showing that the generating function is in fact smooth. We give below further remarks on that.

In order to prove Property (b), i.e., Theorem 3.3 (Theorem 3.1 being proven), we rewrite the second derivative of the generating function, which is the thermodynamic limit of the quantum fluctuations of currents (Theorem 3.1 (i)), as a trace of some explicit positive operator in the one-particle Hilbert space. This quantity can be estimated from below by the Hilbert-Schmidt norm of a kind of current observable in the one-particle Hilbert space. Various computations and estimates then imply Theorem 3.3 .

As discussed in [1], observe the existence of a large mathematical literature on charged transport properties of fermions in disordered media, see for instance [20, 21, 22, 23, 24, 25, 26, 27, 28] and references therein. However, it is not the purpose of this introduction to go into the details of the history of this specific research field. For a (non-exhaustive) historical perspective on linear conductivity (Ohm's law), see, e.g., [29] or our previous papers [5, 6, 10, 18, 30, 31, 32].

To conclude, this paper is organized as follows:

- In Section 2, we describe the mathematical framework, which is the one of [1, 6, 18, It refers to quasi-free fermions on the lattice in disordered media. Although all the problem 
can be formulated, in a mathematically equivalent way, in the one-particle (or Hilbert space) setting [1, Appendix C.3], since the underlying physical system is a many-body one, it is conceptually more appropriate to state our results within the algebraic formulation for lattice fermion systems, like in [1, 6, 18. Short complementary discussions on response of quasi-free fermion systems to electric fields can be found in [1, Appendix C].

- In Section 3, the main results are stated. In particular, Property (a) described above refers to Section 3.1, while Property (b) is explained in Section 3.2.

- Section 4 gathers all technical proofs. In particular, Sections 4.1 4.2 give preliminary definitions and observations, while Sections 4.3 and 4.4 refer to the proofs of Theorems 3.1 (i) and 3.3 , respectively.

\section{Notation 1.1}

$A$ norm on a generic vector space $\mathcal{X}$ is denoted by $\|\cdot\|_{\mathcal{X}}$. The Banach space of all bounded linear operators on $\left(\mathcal{X},\|\cdot\|_{\mathcal{X}}\right)$ is denoted by $\mathcal{B}(\mathcal{X})$. The scalar product of any Hilbert space $\mathcal{X}$ is denoted by $\langle\cdot, \cdot\rangle_{\mathcal{X}}$. We use the convention $\mathbb{R}^{+} \doteq\{x \in \mathbb{R}: x>0\}$ while $\mathbb{R}_{0}^{+} \doteq \mathbb{R}^{+} \cup\{0\}$. For any random variable $X, \mathbb{E}[X]$ denotes its expectation and $\operatorname{Var}[X]$ its variance.

\section{Setup of the Problem}

We use the mathematical framework of [1, 6, 18] in order to study fermions on the lattice.

\subsection{Random Tight-Binding Model}

We consider conducting fermions in a cubic crystal represented by the $d$-dimensional cubic lattice $\mathbb{Z}^{d}(d \in \mathbb{N})$. The corresponding one-particle Hilbert space is thus $\mathfrak{h} \doteq \ell^{2}\left(\mathbb{Z}^{d} ; \mathbb{C}\right)$. Its canonical orthonormal basis is denoted by $\left\{\mathfrak{e}_{x}\right\}_{x \in \mathbb{Z}^{d}}$, where $\mathfrak{e}_{x}(y) \doteq \delta_{x, y}$ for all $x, y \in \mathbb{Z}^{d}$. $\left(\delta_{x, y}\right.$ is the Kronecker delta.)

Disorder in the crystal is modeled via a probability space $\left(\Omega, \mathfrak{A}_{\Omega}, \mathfrak{a}_{\Omega}\right)$, defined as follows: Using the sets

$$
\mathbb{D} \doteq\{z \in \mathbb{C}:|z| \leq 1\} \quad \text { and } \quad \mathfrak{b} \doteq\left\{\left\{x, x^{\prime}\right\} \subseteq \mathbb{Z}^{d}:\left|x-x^{\prime}\right|=1\right\}
$$

we define

$$
\Omega \doteq[-1,1]^{\mathbb{Z}^{d}} \times \mathbb{D}^{\mathfrak{b}} \quad \text { and } \quad \mathfrak{A}_{\Omega} \doteq\left(\otimes_{x \in \mathbb{Z}^{d}} \mathfrak{A}_{x}^{(1)}\right) \otimes\left(\otimes_{\mathbf{x} \in \mathfrak{b}} \mathfrak{A}_{\mathbf{x}}^{(2)}\right)
$$

where $\mathfrak{A}_{x}^{(1)}, x \in \mathbb{Z}^{d}$, and $\mathfrak{A}_{\mathbf{x}}^{(2)}, \mathbf{x} \in \mathfrak{b}$, are the Borel $\sigma$-algebras of respectively the interval $[-1,1]$ and the unit disc $\mathbb{D}$, both with respect to their usual metric topology. The distribution $\mathfrak{a}_{\Omega}$ is an ergodic probability measure on the measurable space $\left(\Omega, \mathfrak{A}_{\Omega}\right)$. See [1] for more details. Below, $\mathbb{E}[\cdot]$ and $\operatorname{Var}[\cdot]$ always refer to expectations and variances associated with $\mathfrak{a}_{\Omega}$.

Given $\vartheta \in \mathbb{R}_{0}^{+}$and $\omega=\left(\omega_{1}, \omega_{2}\right) \in \Omega$ we define a bounded self-adjoint operator $\Delta_{\omega, \vartheta} \in \mathcal{B}(\mathfrak{h})$ encoding the hopping amplitudes of a single particle in the lattice:

$$
\begin{aligned}
{\left[\Delta_{\omega, \vartheta}(\psi)\right](x) \doteq } & 2 d \psi(x)-\sum_{j=1}^{d}\left(\left(1+\vartheta \overline{\omega_{2}\left(\left\{x, x-e_{j}\right\}\right)}\right) \psi\left(x-e_{j}\right)\right. \\
& \left.+\psi\left(x+e_{j}\right)\left(1+\vartheta \omega_{2}\left(\left\{x, x+e_{j}\right\}\right)\right)\right)
\end{aligned}
$$

for any $x \in \mathbb{Z}^{d}$ and $\psi \in \mathfrak{h}$, where $\left\{e_{k}\right\}_{k=1}^{d}$ is the canonical basis of $\mathbb{R}^{d}$. If $\vartheta=0, \Delta_{\omega, 0}$ is (up to a minus sign) the usual $d$-dimensional discrete Laplacian. Random (electromagnetic) vector 
potentials can also be implemented in our model, since $\omega_{2}$ takes values in the unit disc $\mathbb{D} \subseteq \mathbb{C}$. Then, the random tight-binding model is the one-particle Hamiltonian defined by

$$
h^{(\omega)} \doteq \Delta_{\omega, \vartheta}+\lambda \omega_{1}, \quad \omega=\left(\omega_{1}, \omega_{2}\right) \in \Omega, \lambda, \vartheta \in \mathbb{R}_{0}^{+},
$$

where the function $\omega_{1}: \mathbb{Z}^{d} \rightarrow[-1,1]$ is identified with the corresponding (self-adjoint) multiplication operator. The celebrated tight-binding Anderson model corresponds to the special case $\vartheta=0$.

\section{$2.2 C^{*}$-Algebraic Setting}

We denote by $\mathcal{U}$ the universal unital $C^{*}$-algebra generated by elements $\{a(\psi)\}_{\psi \in \mathfrak{h}}$ satisfying the canonical anticommutation relations (CAR): For all $\psi, \varphi \in \mathfrak{h}$,

$$
a(\psi) a(\varphi)=-a(\varphi) a(\psi), \quad a(\psi) a(\varphi)^{*}+a(\varphi)^{*} a(\psi)=\langle\psi, \varphi\rangle_{\mathfrak{h}} \mathbf{l} .
$$

As is usual, $a(\psi)$ and $a(\psi)^{*}$ refer to, respectively, annihilation and creation operators in the fermionic Fock space representation.

For all $\omega \in \Omega$ and $\lambda, \vartheta \in \mathbb{R}_{0}^{+}$, a dynamics on the $C^{*}$-algebra $\mathcal{U}$ is defined by the unique strongly continuous group $\tau^{(\omega)} \doteq\left(\tau_{t}^{(\omega)}\right)_{t \in \mathbb{R}}$ of (Bogoliubov) $*$-automorphisms of $\mathcal{U}$ satisfying

$$
\tau_{t}^{(\omega)}(a(\psi))=a\left(\mathrm{e}^{i t h^{(\omega)}} \psi\right), \quad t \in \mathbb{R}, \psi \in \mathfrak{h} .
$$

See (3) as well as [33, Theorem 5.2.5] for more details on Bogoliubov automorphisms.

For any realization $\omega \in \Omega$ and disorder strengths $\lambda, \vartheta \in \mathbb{R}_{0}^{+}$, the thermal equilibrium state of the system at inverse temperature $\beta \in \mathbb{R}^{+}$(i.e., $\beta>0$ ) is by definition the unique $\left(\tau^{(\omega)}, \beta\right)$ KMS state $\varrho^{(\omega)}$, see [33, Example 5.3.2.] or [34, Theorem 5.9]. It is well-known that such a state is stationary with respect to the dynamics $\tau^{(\omega)}$, that is,

$$
\varrho^{(\omega)} \circ \tau_{t}^{(\omega)}=\varrho^{(\omega)}, \quad \omega \in \Omega, t \in \mathbb{R} .
$$

The state $\varrho^{(\omega)}$ is also gauge-invariant, quasi-free and satisfies

$$
\varrho^{(\omega)}\left(a^{*}(\varphi) a(\psi)\right)=\left\langle\psi, \frac{1}{1+\mathrm{e}^{\beta h^{(\omega)}}} \varphi\right\rangle_{\mathfrak{h}}, \quad \varphi, \psi \in \mathfrak{h} .
$$

The gauge-invariant quasi-free state with two-point correlation functions given by $(6)$ for $\beta=0$ is the tracial state (or chaotic state), denoted by $\operatorname{tr} \in \mathcal{U}^{*}$.

Recall that gauge-invariant quasi-free states are positive linear functionals $\rho \in \mathcal{U}^{*}$ such that $\rho(\mathbf{l})=1$ and, for all $N_{1}, N_{2} \in \mathbb{N}$ and $\psi_{1}, \ldots, \psi_{N_{1}+N_{2}} \in \mathfrak{h}$,

$$
\rho\left(a^{*}\left(\psi_{1}\right) \cdots a^{*}\left(\psi_{N_{1}}\right) a\left(\psi_{N_{1}+N_{2}}\right) \cdots a\left(\psi_{N_{1}+1}\right)\right)=0
$$

if $N_{1} \neq N_{2}$, while in the case $N_{1}=N_{2} \equiv N$,

$$
\rho\left(a^{*}\left(\psi_{1}\right) \cdots a^{*}\left(\psi_{N}\right) a\left(\psi_{2 N}\right) \cdots a\left(\psi_{N+1}\right)\right)=\operatorname{det}\left[\rho\left(a^{*}\left(\psi_{k}\right) a\left(\psi_{N+l}\right)\right)\right]_{k, l=1}^{N} .
$$

See, e.g., [35, Definition 3.1], which refers to a more general notion of quasi-free states. The gauge-invariant property corresponds to Equation (7) whereas [35, Definition 3.1, Condition (3.1)] only imposes the quasi-free state to be even, which is a strictly weaker property than being gauge-invariant. 


\subsection{Linear Response Current Density}

(i) Paramagnetic currents: Fix $\omega \in \Omega$ and $\vartheta \in \mathbb{R}_{0}^{+}$. For any oriented edge $(x, y) \in\left(\mathbb{Z}^{d}\right)^{2}$, we define the paramagneti ${ }^{2}$ current observable by

$$
I_{(x, y)}^{(\omega)} \doteq-2 \Im \mathrm{m}\left(\left\langle\mathfrak{e}_{x}, \Delta_{\omega, \vartheta} \mathfrak{e}_{y}\right\rangle_{\mathfrak{h}} a\left(\mathfrak{e}_{x}\right)^{*} a\left(\mathfrak{e}_{y}\right)\right),
$$

where, as is usual, the real and imaginary parts of any element $A \in \mathcal{U}$ are respectively defined by

$$
\Re \mathrm{e}(A) \doteq \frac{1}{2}\left(A+A^{*}\right) \quad \text { and } \quad \Im \mathrm{m}(A) \doteq \frac{1}{2 i}\left(A-A^{*}\right) .
$$

The self-adjoint elements $I_{(x, y)}^{(\omega)} \in \mathcal{U}$ are seen as current observables because they satisfy a discrete continuity equation, as explained in [1, Appendix C]. This "second-quantized" definition of a current observable and the usual one in the one-particle setting, like in [20, 22, 23], are perfectly equivalent, in the case of non-interacting fermions. See for instance [1, Appendix C.3].

(ii) Conductivity: As is usual, $[A, B] \doteq A B-B A \in \mathcal{U}$ denotes the commutator between the elements $A \in \mathcal{U}$ and $B \in \mathcal{U}$. For any finite subset $\Lambda \subsetneq \mathbb{Z}^{d}$, we define the space-averaged transport coefficient observable $\mathcal{C}_{\Lambda}^{(\omega)} \in C^{1}\left(\mathbb{R} ; \mathcal{B}\left(\mathbb{R}^{d} ; \mathcal{U}^{d}\right)\right)$, with respect to the canonical basis $\left\{e_{q}\right\}_{q=1}^{d}$ of $\mathbb{R}^{d}$, by the corresponding matrix entries

$$
\begin{aligned}
\left\{\mathcal{C}_{\Lambda}^{(\omega)}(t)\right\}_{k, q} \doteq & \frac{1}{|\Lambda|} \sum_{x, y, x+e_{k}, y+e_{q} \in \Lambda} \int_{0}^{t} i\left[\tau_{-\alpha}^{(\omega)}\left(I_{\left(y+e_{q}, y\right)}^{(\omega)}\right), I_{\left(x+e_{k}, x\right)}^{(\omega)}\right] \mathrm{d} \alpha \\
& +\frac{2 \delta_{k, q}}{|\Lambda|} \sum_{x \in \Lambda} \Re \mathrm{e}\left(\left\langle\mathfrak{e}_{x+e_{k}}, \Delta_{\omega, \vartheta} \mathfrak{e}_{x}\right\rangle a\left(\mathfrak{e}_{x+e_{k}}\right)^{*} a\left(\mathfrak{e}_{x}\right)\right)
\end{aligned}
$$

for any $\omega \in \Omega, t \in \mathbb{R}, \lambda, \vartheta \in \mathbb{R}_{0}^{+}$and $k, q \in\{1, \ldots, d\}$. It is the conductivity observable matrix associated with the lattice region $\Lambda$ and time $t$. See [1, Appendix C]. In fact, the first term in the right-hand side of (11) corresponds to the paramagnetic coefficient, whereas the second one is the diamagnetic component. For more details, see [32, Theorem 3.7].

(iii) Linear response current density: Fix a direction $\vec{w} \in \mathbb{R}^{d}$ with $\|\vec{w}\|_{\mathbb{R}^{d}}=1$ and a (timedependent) continuous, compactly supported, electric field $\mathcal{E} \in C_{0}^{0}\left(\mathbb{R} ; \mathbb{R}^{d}\right)$, i.e., the external electric field is a continuous function $t \mapsto \mathcal{E}(t) \in \mathbb{R}^{d}$ of time $t \in \mathbb{R}$, with compact support. Then, as it is explained in [1, Appendix C] as well as in [6, 32 $]^{3}$, the space-averaged linear response current observable in the lattice region $\Lambda$ and at time $t=0$ in the direction $\vec{w}$ is equal to

$$
\mathbb{I}_{\Lambda}^{(\omega, \mathcal{E})} \doteq \sum_{k, q=1}^{d} w_{k} \int_{-\infty}^{0}\{\mathcal{E}(\alpha)\}_{q}\left\{\mathcal{C}_{\Lambda}^{(\omega)}(-\alpha)\right\}_{k, q} \mathrm{~d} \alpha
$$

By [5, 6], the macroscopic (linear response) current density produced by electric fields $\mathcal{E} \in$ $C_{0}^{0}\left(\mathbb{R} ; \mathbb{R}^{d}\right)$ at time $t=0$ in the direction $\vec{w}$ is consequently equal to

$$
x^{(\mathcal{E})} \doteq \lim _{L \rightarrow \infty} \mathbb{E}\left[\varrho^{(\cdot)}\left(\mathbb{I}_{\Lambda_{L}^{(\cdot \mathcal{E})}}\right)\right] \in \mathbb{R}
$$

\footnotetext{
${ }^{2}$ Diamagnetic currents correspond to the ballistic movement of charged particles driven by electric fields. Their presence leads to the progressive appearance of paramagnetic currents which are responsible for heat production. For more details, see [6, 31, 32] as well as [1, Appendix C] on linear response currents.

${ }^{3}$ Strictly speaking, these papers use smooth electric fields, but the extension to the continuous case is straightforward.
} 
where $\Lambda_{L} \doteq\{\mathbb{Z} \cap[-L, L]\}^{d}$ for any $L \in \mathbb{R}_{0}^{+}$. In order to obtain the current density at any time $t \in \mathbb{R}$ in the direction $\vec{w}$, it suffices to replace $\mathcal{E} \in C_{0}^{0}\left(\mathbb{R} ; \mathbb{R}^{d}\right)$ in the last two equations with

$$
\mathcal{E}_{t}(\alpha) \doteq \mathcal{E}(\alpha+t), \quad \alpha \in \mathbb{R}
$$

For a short summary on response of quasi-free fermion systems to electric fields, see [1, Appendix $\mathrm{C}]$.

\subsection{Large Deviations for Microscopic Current Densities}

Fix again a direction $\vec{w} \in \mathbb{R}^{d}$ with $\|\vec{w}\|_{\mathbb{R}^{d}}=1$ and a time-dependent electric field $\mathcal{E} \in C_{0}^{0}\left(\mathbb{R} ; \mathbb{R}^{d}\right)$. Recall that $\Lambda_{L} \doteq\{\mathbb{Z} \cap[-L, L]\}^{d}$ for any $L \in \mathbb{R}_{0}^{+}$. From [5, 6] combined with [1, Corollary 3.2], it follows that the distributions $4^{4}$ of the microscopic current density observables $\left(\mathbb{I}_{\Lambda_{L}}^{(\omega, \mathcal{E})}\right)_{L \in \mathbb{R}^{+}}$, in the state $\varrho^{(\omega)}$, weak* converge, for $\omega \in \Omega$ almost surely, to the delta distribution at the macroscopic value $x^{(\mathcal{E})}$, well-defined by Equation (13). By [1, Corollary 3.5], the quantum uncertainty around the macroscopic value disappears exponentially fast, as $L \rightarrow \infty$.

To arrive at that conclusion we use in [1] the large deviation formalism for the microscopic (linear response) current density in the state $\varrho^{(\omega)}$. More precisely, we prove in [1, Corollary 3.2] that, almost surely $\left.\right|^{5}$ (or with probability one in $\Omega$ ), for any borel subset $\mathcal{G}$ of $\mathbb{R}$ with interior and closure respectively denoted by $\mathcal{G}^{\circ}$ and $\overline{\mathcal{G}}$,

$$
\begin{aligned}
-\inf _{x \in \mathcal{G}^{\circ}} \mathrm{I}^{(\mathcal{E})}(x) \leq \liminf _{L \rightarrow \infty} \frac{1}{\left|\Lambda_{L}\right|} \ln \varrho^{(\omega)} & \left(\mathbf{1}\left[\mathbb{I}_{\Lambda_{L}}^{(\omega, \mathcal{E})} \in \mathcal{G}\right]\right) \\
& \leq \limsup _{L \rightarrow \infty} \frac{1}{\left|\Lambda_{L}\right|} \ln \varrho^{(\omega)}\left(\mathbf{1}\left[\mathbb{I}_{\Lambda_{L}}^{(\omega, \mathcal{E})} \in \mathcal{G}\right]\right) \leq-\inf _{x \in \mathcal{G}} \mathrm{I}^{(\mathcal{E})}(x) .
\end{aligned}
$$

By an abuse of notation ${ }^{6}$, we applied above the (non-continuous) characteristic function $\mathbf{1}[x \in \mathcal{G}]$ to $\mathbb{I}_{\Lambda_{L}}^{(\omega, \mathcal{E})}$. Here, by [1, Theorems 3.1, 3.4, Corollary 3.2], the so-called good ${ }^{7}$ rate function $\mathrm{I}^{(\mathcal{E})}$ is a deterministic, positive, lower-semicontinuous, convex function defined by

$$
\mathrm{I}^{(\mathcal{E})}(x) \doteq \sup _{s \in \mathbb{R}}\left\{s x-\mathrm{J}^{(s \mathcal{E})}\right\} \geq 0, \quad x \in \mathbb{R}
$$

where

$$
\mathrm{J}^{(\mathcal{E})} \doteq \lim _{L \rightarrow \infty} \frac{1}{\left|\Lambda_{L}\right|} \mathbb{E}\left[\ln \varrho^{(\cdot)}\left(\mathrm{e}^{\left.\left|\Lambda_{L}\right| \mathbb{I}_{\Lambda_{L}^{(\cdot, \mathcal{E})}}^{(}\right)}\right)\right] \in \mathbb{R}
$$

for all $\beta \in \mathbb{R}^{+}, \vartheta, \lambda \in \mathbb{R}_{0}^{+}, \mathcal{E} \in C_{0}^{0}\left(\mathbb{R} ; \mathbb{R}^{d}\right)$ and $\vec{w} \in \mathbb{R}^{d}$ with $\|\vec{w}\|_{\mathbb{R}^{d}}=1$. By [1, Theorem 3.4], I $\mathrm{I}^{(\mathcal{E})}$ restricted to the interior of its domain is continuous and, as clearly expected, the rate function $\mathrm{I}^{(\mathcal{E})}$ vanishes on the macroscopic (linear response) current density $x^{(\mathcal{E})}$, i.e., $\mathrm{I}^{(\mathcal{E})}\left(x^{(\mathcal{E})}\right)=0$, whereas $\mathrm{I}^{(\mathcal{E})}(x)>0$ for all $x \neq x^{(\mathcal{E})}$.

\footnotetext{
${ }^{4}$ Here, like in [1, the distribution associated to a selfadjoint element $A$ of a unital $C^{*}$-algebra $\mathfrak{A}$ and to a state on this algebra is the probability measure on the spectrum of $A$ representing the restriction of the state to the unital $C^{*}$-subalgebra of $\mathfrak{A}$ generated by $A$. Recall that this measure exists and is unique, by the Riesz-Markov representation theorem.

${ }^{5}$ The measurable subset $\tilde{\Omega} \subseteq \Omega$ of full measure of [1, Corollary 3.2] does not depend on $\beta \in \mathbb{R}^{+}, \vartheta, \lambda \in \mathbb{R}_{0}^{+}$, $\mathcal{E} \in C_{0}^{0}\left(\mathbb{R} ; \mathbb{R}^{d}\right)$ and $\vec{w} \in \mathbb{R}^{d}$ with $\|\vec{w}\|_{\mathbb{R}^{d}}=1$.

${ }^{6}$ In fact, the object $\varrho^{(\omega)}\left(\mathbf{1}\left[\mathbb{I}_{\Lambda_{L}}^{(\omega, \mathcal{E})} \in \mathcal{G}\right]\right)$ can be easily given a precise mathematical sense by using the (up to unitary equivalence) unique cyclic representation of the $C^{*}$-algebra $\mathcal{U}$ associated to the state $\varrho^{(\omega)}$, noting that the bicommutant of a $*$-algebra in any representation is a von Neumann algebra and thus admits a mesurable calculus.

${ }^{7}$ It means, in this context, that $\left\{x \in \mathbb{R}: \mathrm{I}^{(\mathcal{E})}(x) \leq m\right\}$ is compact for any $m \geq 0$.
} 
For any $\mathcal{E} \in C_{0}^{0}\left(\mathbb{R} ; \mathbb{R}^{d}\right)$, note that Equation 15$)$ means that $\mathrm{I}^{(\mathcal{E})}$ is the Legendre-Fenchel transform of the generating function $s \mapsto \mathrm{J}^{(s \mathcal{E})}$ from $\mathbb{R}$ to itself, which is a well-defined, continuously differentiable, convex function, by [1, Theorem 3.1]. Moreover, by [1, Corollary 4.20 and Equation (54)], for any $\beta \in \mathbb{R}^{+}, \vartheta, \lambda \in \mathbb{R}_{0}^{+}, \mathcal{E} \in C_{0}^{0}\left(\mathbb{R} ; \mathbb{R}^{d}\right), \vec{w} \in \mathbb{R}^{d}$ with $\|\vec{w}\|_{\mathbb{R}^{d}}=1$, the macroscopic current density defined by 13 can be expressed in terms of the generating function:

$$
x^{(\mathcal{E})}=\left.\partial_{s} \mathrm{~J}^{(s \mathcal{E})}\right|_{s=0} \text {. }
$$

\section{Main Results}

In order to provide a rather complete study of conductivity at the atomic scale for free-fermions in a lattice, we analyse here the rate function defined by Equation (15) in much more detail than in [1]. See [1, Corollary 3.2]. We focus on the behavior of the rate function near the macroscopic value of the current density (see (17)), because it establishes a very interesting connection between exponential suppression of quantum uncertainties at the atomic scale and the concept of quantum fluctuations, in the case of currents.

\subsection{Quantum Fluctuations of Linear Response Currents and Rate Function}

For any inverse temperature $\beta \in \mathbb{R}^{+}$, disorder strengths $\vartheta, \lambda \in \mathbb{R}_{0}^{+}$, disorder realization $\omega \in \Omega$, direction $\vec{w} \in \mathbb{R}^{d}$ with $\|\vec{w}\|_{\mathbb{R}^{d}}=1$ and time-dependent electric field $\mathcal{E} \in C_{0}^{0}\left(\mathbb{R} ; \mathbb{R}^{d}\right)$, the quantum fluctuations of linear response currents in cubic boxes are defined to be

$$
\mathbf{F}_{L}^{(\omega, \mathcal{E})} \doteq\left|\Lambda_{L}\right|\left(\varrho^{(\omega)}\left(\left(\mathbb{I}_{\Lambda_{L}}^{(\omega, \mathcal{E})}\right)^{2}\right)-\varrho^{(\omega)}\left(\mathbb{I}_{\Lambda_{L}}^{(\omega, \mathcal{E})}\right)^{2}\right) \geq 0, \quad L \in \mathbb{R}_{0}^{+},
$$

with $\Lambda_{L} \doteq\{\mathbb{Z} \cap[-L, L]\}^{d}$ and $\mathbb{I}_{\Lambda_{L}}^{(\omega, \mathcal{E})}(t)$ being the space-averaged linear response current defined by 12 . Observe that

$$
\left|\Lambda_{L}\right| \varrho^{(\omega)}\left(\mathbb{I}_{\Lambda_{L}}^{(\omega, \mathcal{E})}(t)\right), \quad L \in \mathbb{R}_{0}^{+},
$$

is the (total) current linear response (in the direction $\vec{w}$ ) to the electric field and, consequently,

$$
\mathbf{F}_{L}^{(\omega, \mathcal{E})}=\frac{1}{\left|\Lambda_{L}\right|}\left(\varrho^{(\omega)}\left(\left(\left|\Lambda_{L}\right| \mathbb{I}_{\Lambda_{L}}^{(\omega, \mathcal{E})}\right)^{2}\right)-\varrho^{(\omega)}\left(\left|\Lambda_{L}\right| \mathbb{I}_{\Lambda_{L}}^{(\omega, \mathcal{E})}\right)^{2}\right), \quad L \in \mathbb{R}_{0}^{+},
$$

are naturally seen as (normal) quantum fluctuations of the (total) linear response current. Note that these quantum fluctuations are not quite the same current fluctuations of [5, 10], which correspond only to the paramagnetic component of the current, whereas $\left(\mathbf{F}_{L}^{(\omega, \mathcal{E})}\right)$ also includes the diamagnetic one and thus refers to the total current.

Recall that $x^{(\mathcal{E})}$ is the macroscopic (linear response) current density defined by $(13)$ and $\mathrm{I}^{(\mathcal{E})}$ (15) is the (good) rate function associated with the large deviation principle of the sequence $\left\{\mathbb{I}_{\Lambda_{L}(\omega, \mathcal{E})}\right\}_{L \in \mathbb{R}^{+}}$of microscopic current densities, in the KMS state $\varrho^{(\omega)}$ and with speed $\left|\Lambda_{L}\right|$. See, e.g., [1, Theorems 3.1, 3.4, Corollary 3.2]. We are now in a position to connect the quantum fluctuations of (linear response) currents with the generating and rate functions associated with the large deviation principle of microscopic current densities.

\section{Theorem 3.1 (Quantum fluctuations and rate function)}

There is a measurable subset $\tilde{\Omega} \subseteq \Omega$ of full measure such that, for all $\beta \in \mathbb{R}^{+}, \vartheta, \lambda \in \mathbb{R}_{0}^{+}$, 
$\omega \in \tilde{\Omega}, \mathcal{E} \in C_{0}^{0}\left(\mathbb{R} ; \mathbb{R}^{d}\right)$ and $\vec{w} \in \mathbb{R}^{d}$ with $\|\vec{w}\|_{\mathbb{R}^{d}}=1$, the following properties hold true:

(i) The generating function $s \mapsto \mathrm{J}^{(s \mathcal{E})}$ defined by $(16)$ belongs to $C^{\infty}(\mathbb{R} ; \mathbb{R})$ and satisfies

$$
\left.\partial_{s}^{2} \mathrm{~J}^{(s \mathcal{E})}\right|_{s=0}=\lim _{L \rightarrow \infty} \mathbb{E}\left[\mathbf{F}_{L}^{(\cdot, \mathcal{E})}\right]=\lim _{L \rightarrow \infty} \mathbf{F}_{L}^{(\omega, \mathcal{E})} \geq 0 .
$$

(ii) The rate function $\mathrm{I}^{(\mathcal{E})}$ satisfies the asymptotics

$$
\mathrm{I}^{(\mathcal{E})}(x)=\frac{1}{\left.2 \partial_{s}^{2} \mathrm{~J}^{(\mathcal{E})}\right|_{s=0}}\left(x-x^{(\mathcal{E})}\right)^{2}+o\left(\left(x-x^{(\mathcal{E})}\right)^{2}\right),
$$

provided that $\left.\partial_{s}^{2} \mathrm{~J}^{(s \mathcal{E})}\right|_{s=0} \neq 0$.

Proof. Fix all parameters of the theorem. By Corollary 4.2 , the generating function $s \mapsto \mathrm{J}^{(s \mathcal{E})}$ belongs to $C^{2}(\mathbb{R} ; \mathbb{R})$ and satisfies $(20)$. As explained after Corollary 4.2 , under the assumptions of Theorem 3.1, one can straightforwardly extend our arguments to prove that the generating function $s \mapsto \mathrm{J}^{(s \mathcal{E})}$ defined by 16 is infinitely differentiable. (i) thus holds true. It remains to prove Assertion (ii): Since the map $s \mapsto \mathrm{J}^{(s \mathcal{E})}$ from $\mathbb{R}$ to itself is convex and belongs (at least) to $C^{1}(\mathbb{R} ; \mathbb{R})$ (see, e.g., Assertion (i) or [1, Theorem 3.1]), all finite solutions $s(x) \in \mathbb{R}$ of the variational problem 15 for $x \in \mathbb{R}$, i.e.,

$$
\mathrm{I}^{(\mathcal{E})}(x)=s(x) x-\mathrm{J}^{(s(x) \mathcal{E})},
$$

satisfy

$$
x=f(s(x)),
$$

with $f$ being the real-valued function defined by

$$
f(s) \doteq \partial_{s} \mathrm{~J}^{(s \mathcal{E})}, \quad s \in \mathbb{R} .
$$

Assume now that $\left.\partial_{s}^{2} \mathrm{~J}^{(s \mathcal{E})}\right|_{s=0} \neq 0$, which is equivalent in this case to

$$
\partial_{s} f(0)=\left.\partial_{s}^{2} \mathrm{~J}^{(s \mathcal{E})}\right|_{s=0}>0,
$$

by positivity of fluctuations (see (i)). Since, by Corollary 4.2 , the mapping $s \mapsto \mathrm{J}^{(s \mathcal{E})}$ from $\mathbb{R}$ to itself belongs (at least) to $C^{2}(\mathbb{R} ; \mathbb{R})$, by the inverse function theorem combined with $(21)-(24)$ and $(17)$, there is an open interval

$$
\mathcal{I} \subseteq\left\{f(s): s \in \mathbb{R} \text { such that } \partial_{s} f(s)>0\right\} \subseteq \mathbb{R}
$$

containing $x^{(\mathcal{E})}=f(0)$ and a $C^{1}$-function $x \mapsto s(x)$ from $\mathcal{I}$ to $\mathbb{R}$ such that Equations (21)-(23) hold true. In particular,

$$
\partial_{s} f(s(x))=\left.\partial_{s}^{2} \mathrm{~J}^{(s \mathcal{E})}\right|_{s=s(x)}>0, \quad x \in \mathcal{I} .
$$

Clearly,

$$
\partial_{x} s(x)=\frac{1}{\partial_{s} f(s(x))}, \quad x \in \mathcal{I}
$$

We thus infer from (21)-(23) and (26), together with (i), that

$$
\partial_{x} \mathrm{I}^{(\mathcal{E})}(x)=s(x), \quad x \in \mathcal{I} .
$$

Consequently, $\partial_{x} \mathrm{I}^{(\mathcal{E})}$ is differentiable on $\mathcal{I}$ with derivative given by

$$
\partial_{x}^{2} \mathrm{I}^{(\mathcal{E})}(x)=\partial_{x} s(x), \quad x \in \mathcal{I} .
$$


As a consequence, $\mathrm{I}^{(\mathcal{E})}$ is twice differentiable on $\mathcal{I} \supseteq\left\{x^{(\mathcal{E})}\right\}$ and, using the Taylor theorem at the point $x^{(\mathcal{E})}$, one obtains that

$$
\mathrm{I}^{(\mathcal{E})}(x)=s\left(x^{(\mathcal{E})}\right)\left(x-x^{(\mathcal{E})}\right)+\frac{1}{2} \partial_{x} s\left(x^{(\mathcal{E})}\right)\left(x-x^{(\mathcal{E})}\right)^{2}+o\left(\left(x-x^{(\mathcal{E})}\right)^{2}\right),
$$

provided (24) holds true. Since, by (17), 23) and $(26), s\left(x^{(\mathcal{E})}\right)=0$ and

$$
\partial_{x} s\left(x^{(\mathcal{E})}\right)=\frac{1}{\partial_{s} f(0)}=\left.\frac{1}{\partial_{s}^{2} \mathrm{~J}^{(s \mathcal{E})}}\right|_{s=0}
$$

one thus deduces (ii) from (27).

This theorem is a very interesting observation on the physics of fermionic systems because it shows that the experimental measure of the rate function of currents around the expected value leads to an experimental estimate on the corresponding quantum fluctuations. Conversely, by Theorem 3.1, an experimental estimate on these quantum fluctuations gives the behavior of the corresponding rate function around the expected value. This phenomenon is certainly not restricted to fermionic currents and this is a new observation on transport properties of quantum many-body systems, to our knowledge.

\section{Remark 3.2 (Extension of Theorem 3.1)}

The proof of Theorem 3.1 can be generalized to very general kinetic terms (i.e., it does not really depend on the special choice $\left.\Delta_{\omega, \vartheta}\right)$, provided the pivotal Combes-Thomas estimate holds true for the one-particle Hamiltonian. Note, however, that this would require a new, more complicated, definition of currents, which results from the commutator of the density operator at fixed lattice site with the kinetic term (cf. continuity equations on the CAR algebra [32, Eqs. (38)-(39)]). We did not implement this generalization here, because we think that, conceptually, the gain is too small as compared to the drawbacks concerning notations, definitions, and technical proofs. Instead, we aim at obtaining an extension of Theorem 3.1 to weakly interacting fermionic systems by using new constructive methods based on Grassmann-Berezin integrals, BrydgesKennedy expansions, etc.

\subsection{Non-Vanishing Quantum Fluctuations of Linear Response Cur- rents}

By Theorem 3.1, the behavior of the rate function within a neighborhood of the macroscopic current densities is directly related to the quantum fluctuations of the linear response current, provided these fluctuations do not vanish in the thermodynamic limit, i.e., if $\left.\partial_{s}^{2} \mathrm{~J}^{(s \mathcal{E})}\right|_{s=0} \neq 0$ (see Theorem 3.1 (i)). We do not expect this situation to appear in presence of disorder. We discuss this issue in Section 4.4, where we give sufficient conditions ensuring non-vanishing quantum fluctuations of linear response currents in the thermodynamic limit. This study leads to the following theorem:

\section{Theorem 3.3 (Sufficient conditions for non-zero quantum fluctuations)}

Take $\vartheta, \lambda \in \mathbb{R}_{0}^{+}, T, \beta \in \mathbb{R}^{+}, \mathcal{E} \in C_{0}^{0}\left(\mathbb{R} ; \mathbb{R}^{d}\right)$ with support in $[-T, 0]$ and $\vec{w} \doteq\left(w_{1}, \ldots, w_{d}\right) \in$ $\mathbb{R}^{d}$ with $\|\vec{w}\|_{\mathbb{R}^{d}}=1$. Assume that the random variables $\left\{\omega_{1}(z)\right\}_{z \in \mathbb{Z}^{d}}$ are independently and identically distributed (i.i.d.). Then, for sufficiently small $T$ and $\vartheta$,

$$
\left.\partial_{s}^{2} \mathrm{~J}^{(s \mathcal{E})}\right|_{s=0} \geq \frac{\lambda^{2} \Upsilon^{(\mathcal{E}, \vec{w})}}{\left(1+\mathrm{e}^{\beta(2 d(2+\vartheta)+\lambda)}\right)^{2}} \operatorname{Var}\left[(\cdot)_{1}(0)\right]
$$


with

$$
\Upsilon^{(\mathcal{E}, \vec{w})} \doteq\left(\int_{-\infty}^{0}\langle w, \mathcal{E}(\alpha)\rangle_{\mathbb{R}^{d}} \alpha^{2} \mathrm{~d} \alpha\right)^{2}+\frac{1}{2} \sum_{k=1}^{d}\left(w_{k} \int_{-\infty}^{0}(\mathcal{E}(\alpha))_{k} \alpha^{2} \mathrm{~d} \alpha\right)^{2}
$$

In particular, $\left.\partial_{s}^{2} \mathrm{~J}^{(s \mathcal{E})}\right|_{s=0} \neq 0$ whenever $\Upsilon^{(\mathcal{E}, \vec{w})}>0, \omega_{1}(0)$ is not almost surely constant (and thus $\operatorname{Var}\left[(\cdot)_{1}(0)\right]>0$, by Chebychev's inequality) and $T, \vartheta$ are sufficiently small.

Proof. This is a direct consequence of Equations (66) and (68), in Section 4 .

By Theorems 3.1 and 3.3, we thus demonstrate that, in general, the quantum fluctuations of linear response currents do not vanish in the thermodynamic limit and the quantum uncertainty around the macroscopic current density $x^{(\mathcal{E})}$ disappears exponentially fast, as the volume of the cubic box $\Lambda_{L}$ grows, with a rate proportional to the squared deviation of the current from $x^{(\mathcal{E})}$ and the inverse current fluctuation. In particular, by combining Theorem 3.1 (i) with Theorem 3.3 we can obtain an explicit upper bound on the rate function $\mathrm{I}^{(\mathcal{E})}$ around $x^{(\mathcal{E})}$.

The fact that the random variables $\left\{\omega_{1}(z)\right\}_{z \in \mathbb{Z}^{d}}$ are independently and identically distributed (i.i.d.) in Theorem 3.3 is not essential here: For any $\omega \in \Omega$, let $w^{(\omega)} \doteq\left(w_{1}^{(\omega)}, \ldots, w_{d}^{(\omega)}\right) \in$ $\mathbb{R}^{d}$ be the random vector defined by

$$
w_{k}^{(\omega)} \doteq\left(2 \omega_{1}(0)-\omega_{1}\left(e_{k}\right)-\omega_{1}\left(-e_{k}\right)\right) w_{k}, \quad k \in\{1, \ldots, d\}
$$

with $\left\{e_{k}\right\}_{k=1}^{d}$ being the canonical basis of $\mathbb{R}^{d}$. By (64), 66 and (67), it suffices that

$$
\mathbb{E}\left[\left|\int_{-\infty}^{0}\left\langle w^{(\cdot)}, \mathcal{E}(\alpha)\right\rangle_{\mathbb{R}^{d}} \alpha^{2} \mathrm{~d} \alpha\right|^{2}\right]=\operatorname{Var}\left[\int_{-\infty}^{0}\left\langle w^{(\cdot)}, \mathcal{E}(\alpha)\right\rangle_{\mathbb{R}^{d}} \alpha^{2} \mathrm{~d} \alpha\right]>0
$$

in order to ensure non-vanishing quantum fluctuations of linear response currents in the thermodynamic limit, i.e., $\left.\partial_{s}^{2} \mathrm{~J}^{(s \mathcal{E})}\right|_{s=0} \neq 0$.

Theorem 3.3 can be applied to the celebrated tight-binding Anderson model, which corresponds to the special case $\vartheta=0$. This is why we focus on this important example in this theorem. The remaining case of larger parameters $\vartheta, T \in \mathbb{R}_{0}^{+}$can certainly be studied, even if this is not done here.

\section{Technical Proofs}

\subsection{Quasi-Free Fermions in Subregions of the Lattice}

Let $\mathcal{P}_{\mathrm{f}}\left(\mathbb{Z}^{d}\right) \subseteq 2^{\mathbb{Z}^{d}}$ be the set of all non-empty finite subsets of $\mathbb{Z}^{d}$. Like in [1, Section 2.1], we need the sets

$$
\begin{aligned}
\mathfrak{Z} & \doteq\left\{\mathcal{Z} \subseteq 2^{\mathbb{Z}^{d}}:\left(\forall Z_{1}, Z_{2} \in \mathcal{Z}\right) Z_{1} \neq Z_{2} \Rightarrow Z_{1} \cap Z_{2}=\emptyset\right\} \\
\mathfrak{Z}_{\mathrm{f}} & \doteq \mathfrak{Z} \cap\left\{\mathcal{Z} \subseteq \mathcal{P}_{\mathrm{f}}\left(\mathbb{Z}^{d}\right):|\mathcal{Z}|<\infty\right\} .
\end{aligned}
$$

This kind of decomposition over collections of disjoint subsets of the lattice is important to prove Theorem 3.1 (i).

Recall that $\mathfrak{h} \doteq \ell^{2}\left(\mathbb{Z}^{d} ; \mathbb{C}\right)$ and $\mathcal{B}(\mathfrak{h})$ is the Banach space of all bounded linear operators acting on $\mathfrak{h}$. One can restrict the quasi-free dynamics defined by $(5)$ to collections $\mathcal{Z} \in \mathfrak{Z}$ of disjoint subsets of the lattice by using the orthogonal projections $P_{\Lambda}, \Lambda \subseteq \mathbb{Z}^{d}$, defined on the Hilbert space $\mathfrak{h}$ by

$$
\left[P_{\Lambda}(\psi)\right](x) \doteq \begin{cases}\psi(x) & , \text { if } x \in \Lambda \\ 0 & , \text { else }\end{cases}
$$


for any $\psi \in \mathfrak{h}$. Then, the one-particle Hamiltonian within $\mathcal{Z} \in \mathfrak{Z}$ is, by definition, equal to

$$
h_{\mathcal{Z}}^{(\omega)} \doteq \sum_{Z \in \mathcal{Z}} P_{Z} h^{(\omega)} P_{Z} \in \mathcal{B}(\mathfrak{h})
$$

where $h^{(\omega)} \in \mathcal{B}(\mathfrak{h})$ is the random tight-binding model defined by $(3)$ for any $\omega \in \Omega$ and $\lambda, \vartheta \in \mathbb{R}_{0}^{+}$. For any $\mathcal{Z} \in \mathfrak{Z}$, it leads to the unitary group $\left\{\mathrm{e}^{i t h_{\mathcal{Z}}^{(\omega)}}\right\}_{t \in \mathbb{R}}$ acting on the Hilbert space $\mathfrak{h}$.

Similar to Equation (5), for any $\mathcal{Z} \in \mathfrak{Z}$, we consequently define the strongly continuous $\operatorname{group} \tau^{(\omega, \mathcal{Z})} \doteq\left\{\tau_{t}^{(\omega, \mathcal{Z})}\right\}_{t \in \mathbb{R}}$ of Bogoliubov $*$-automorphisms of $\mathcal{U}$ by

$$
\tau_{t}^{(\omega, \mathcal{Z})}(a(\psi))=a\left(\mathrm{e}^{i t h_{\mathcal{Z}}^{(\omega)}} \psi\right), \quad t \in \mathbb{R}, \psi \in \mathfrak{h} .
$$

This corresponds to replace $h^{(\omega)}$ in $(5)$ with $h_{\mathcal{Z}}^{(\omega)}$. Similarly, for any $\mathcal{Z} \in \mathfrak{Z}$, we define the quasi-free state $\varrho_{\mathcal{Z}}^{(\omega)}$ by replacing $h^{(\omega)}$ in Equation $(6)$ with the one-particle Hamiltonian $h_{\mathcal{Z}}^{(\omega)}$ within $\mathcal{Z}$.

If $\mathcal{Z} \in \mathfrak{Z}_{\mathrm{f}}$ then both $\tau^{(\omega, \mathcal{Z})}$ and $\varrho_{\mathcal{Z}}^{(\omega)}$ can be written in terms of bilinear elements 8 , defined as follows: The bilinear element associated with an operator in $C \in \mathcal{B}(\mathfrak{h})$ whose range, $\operatorname{ran}(C)$, is finite dimensional is defined by

$$
\langle\mathrm{A}, C \mathrm{~A}\rangle \doteq \sum_{i, j \in I}\left\langle\psi_{i}, C \psi_{j}\right\rangle_{\mathfrak{h}} a\left(\psi_{i}\right)^{*} a\left(\psi_{j}\right)
$$

where $\left\{\psi_{i}\right\}_{i \in I}$ is any orthonormal basis $\}^{9}$ of a finite dimensional subspace

$$
\mathcal{H} \supseteq \operatorname{ran}(C) \cup \operatorname{ran}\left(C^{*}\right)
$$

of the Hilbert space $\mathfrak{h}$. See [1, Definition 4.3]. For any $\omega \in \Omega$ and $\lambda, \vartheta \in \mathbb{R}_{0}^{+}$, the range of $h_{\mathcal{Z}}^{(\omega)} \in \mathcal{B}(\mathfrak{h})$ is finite-dimensional whenever $\mathcal{Z} \in \mathfrak{Z}_{\mathrm{f}}$ and one checks that, for any time $t \in \mathbb{R}$, inverse temperature $\beta \in \mathbb{R}^{+}$, finite collections $\mathcal{Z} \in \mathfrak{Z}_{\text {f }}$ and elements $B \in \mathcal{U}$,

$$
\tau_{t}^{(\omega, \mathcal{Z})}(B)=\mathrm{e}^{i t\left\langle\mathrm{~A}, h_{\mathcal{Z}}^{(\omega)} \mathrm{A}\right\rangle} B \mathrm{e}^{-i t\left\langle\mathrm{~A}, h_{\mathcal{Z}}^{(\omega)} \mathrm{A}\right\rangle} \quad \text { and } \quad \varrho_{\mathcal{Z}}^{(\omega)}(B)=\frac{\operatorname{tr}\left(B \mathrm{e}^{-\beta\left\langle\mathrm{A}, h_{\mathcal{Z}}^{(\omega)} \mathrm{A}\right\rangle}\right)}{\operatorname{tr}\left(\mathrm{e}^{-\beta\left\langle\mathrm{A}, h_{\mathcal{Z}}^{(\omega)} \mathrm{A}\right\rangle}\right)},
$$

where $\operatorname{tr} \in \mathcal{U}^{*}$ is the tracial state, i.e., the gauge-invariant quasi-free state with two-point correlation functions given by (6) for $\beta=0$. See [1, Equations (27)-(28)]. The dynamics corresponds in this case to the usual dynamics written in the Heisenberg picture of quantum mechanics, while the above quasi-free state is the Gibbs state at inverse temperature $\beta \in \mathbb{R}^{+}$, both associated with the Hamiltonian $\left\langle\mathrm{A}, h_{\mathcal{Z}}^{(\omega)} \mathrm{A}\right\rangle \in \mathcal{U}$ for $\mathcal{Z} \in \mathfrak{Z}_{\mathrm{f}}$.

In order to define the thermodynamic limit, we use the cubic boxes $\Lambda_{\ell} \doteq\{\mathbb{Z} \cap[-\ell, \ell]\}^{d}$ for $\ell \in \mathbb{R}_{0}^{+}$. Then, as $\ell \rightarrow \infty$, for any $t \in \mathbb{R}, \tau_{t}^{\left(\omega,\left\{\Lambda_{\ell}\right\}\right)}$ converges strongly to $\tau_{t}^{(\omega)} \equiv \tau_{t}^{\left(\omega,\left\{\mathbb{Z}^{d}\right\}\right)}$, while $\varrho_{\left\{\Lambda_{\ell}\right\}}^{(\omega)}$ converges in the weak* topology to $\varrho^{(\omega)} \equiv \varrho_{\left\{\mathbb{Z}^{d}\right\}}^{(\omega)}$. For an explicit proof of these well-known facts, see for instance [36, Propositions 3.2.9 and 3.2.13].

\footnotetext{
${ }^{8}$ This refers to the well-known second-quantization of one-particle Hamiltonians in the Fock space representation.

${ }^{9}\langle\mathrm{~A}, C \mathrm{~A}\rangle$ does not depend on the particular choice of $\mathcal{H}$ and its orthonormal basis.
} 


\subsection{Current Observables in Subregions of the Lattice}

Fix once and for all $\vec{w} \in \mathbb{R}^{d}$ with $\|\vec{w}\|_{\mathbb{R}^{d}}=1$. By [1, Equation (29)], for any $\lambda, \vartheta \in \mathbb{R}_{0}^{+}, \omega \in \Omega$, $\mathcal{E} \in C_{0}^{0}\left(\mathbb{R} ; \mathbb{R}^{d}\right), \mathcal{Z} \in \mathfrak{Z}_{\mathrm{f}}$ and $\mathcal{Z}^{(\tau)} \in \mathfrak{Z}$, the linear response current observable is, by definition, equal to

$$
\begin{aligned}
\mathfrak{K}_{\mathcal{Z}, \mathcal{Z}(\tau)}^{(\omega, \mathcal{E})} \doteq & \sum_{k, q=1}^{d} w_{k} \sum_{Z \in \mathcal{Z}} \sum_{x, y, x+e_{k}, y+e_{q} \in Z} \int_{-\infty}^{0}\{\mathcal{E}(\alpha)\}_{q} \mathrm{~d} \alpha \int_{0}^{-\alpha} \mathrm{d} s i\left[\tau_{-s}^{\left(\omega, \mathcal{Z}^{(\tau)}\right)}\left(I_{\left(y+e_{q}, y\right)}^{(\omega)}\right), I_{\left(x+e_{k}, x\right)}^{(\omega)}\right] \\
& +2 \sum_{k=1}^{d} w_{k} \sum_{Z \in \mathcal{Z}} \sum_{x, x+e_{k} \in Z}\left(\int_{-\infty}^{0}\{\mathcal{E}(\alpha)\}_{q} \mathrm{~d} \alpha\right) \Re \mathrm{e}\left(\left\langle\mathfrak{e}_{x+e_{k}}, \Delta_{\omega, \vartheta} \mathfrak{e}_{x}\right\rangle a\left(\mathfrak{e}_{x+e_{k}}\right)^{*} a\left(\mathfrak{e}_{x}\right)\right)
\end{aligned}
$$

with $\left\{e_{k}\right\}_{k=1}^{d}$ being the canonical basis of $\mathbb{R}^{d}$. Recall that $\Re \mathrm{e}(A) \in \mathcal{U}$ is the real part of $A \in \mathcal{U}$, see (10). Note from Equations (11)-(12) that

$$
\mathfrak{K}_{\{\Lambda\},\left\{\mathbb{Z}^{d}\right\}}^{(\omega, \mathcal{E})}=|\Lambda| \mathbb{I}_{\Lambda}^{(\omega, \mathcal{E})}, \quad \Lambda \in \mathcal{P}_{\mathrm{f}}\left(\mathbb{Z}^{d}\right),
$$

are linear response current observables within finite subsets of the lattice.

The above current observables can obviously be rewritten as bilinear elements (30) associated with one-particle operators acting on the Hilbert space $\mathfrak{h}$. In order to give an explicit expression of these operators, we first define, for any $x \in \mathbb{Z}^{d}$, the shift operator $s_{x} \in \mathcal{B}(\mathfrak{h})$ by

$$
\left(s_{x} \psi\right)(y) \doteq \psi(x+y), \quad y \in \mathbb{Z}^{d}, \psi \in \mathfrak{h} .
$$

Note that $s_{x}^{*}=s_{-x}=s_{x}^{-1}$ for any $x \in \mathbb{Z}^{d}$. Then, for every $\omega \in \Omega$ and $\vartheta \in \mathbb{R}_{0}^{+}$, the single-hopping operators are

$$
S_{x, y}^{(\omega)} \doteq\left\langle\mathfrak{e}_{x}, \Delta_{\omega, \vartheta} \mathfrak{e}_{y}\right\rangle_{\mathfrak{h}} P_{\{x\}} s_{x-y} P_{\{y\}}, \quad x, y \in \mathbb{Z}^{d},
$$

where $P_{\{u\}}$ is the orthogonal projection defined by $(28)$ for $\Lambda=\{u\}$ and $u \in \mathbb{Z}^{d}$. Observe that

$$
\left\langle\mathrm{A}, S_{x, y}^{(\omega)} \mathrm{A}\right\rangle=\left\langle\mathfrak{e}_{x}, \Delta_{\omega, \vartheta} \mathfrak{e}_{y}\right\rangle_{\mathfrak{h}} a\left(\mathfrak{e}_{x}\right)^{*} a\left(\mathfrak{e}_{y}\right), \quad x, y \in \mathbb{Z}^{d} .
$$

Similarly, by the identity

$$
\Im \mathrm{m}\{\langle\mathrm{A}, C \mathrm{~A}\rangle\}=\langle\mathrm{A}, \Im \mathrm{m}\{C\} \mathrm{A}\rangle
$$

for any $C \in \mathcal{B}(\mathfrak{h})$ whose range is finite dimensional, the paramagnetic current observables defined by (9) equals

$$
I_{(x, y)}^{(\omega)}=-2\left\langle\mathrm{~A}, \Im \mathrm{m}\left\{S_{x, y}^{(\omega)}\right\} \mathrm{A}\right\rangle, \quad x, y \in \mathbb{Z}^{d},
$$

for each $\omega \in \Omega$ and $\vartheta \in \mathbb{R}_{0}^{+}$. For any $\lambda, \vartheta \in \mathbb{R}_{0}^{+}, \omega \in \Omega, \mathcal{E} \in C_{0}^{0}\left(\mathbb{R} ; \mathbb{R}^{d}\right), \mathcal{Z}^{(\tau)} \in \mathfrak{Z}$ and $\mathcal{Z} \in \mathfrak{Z}_{\mathrm{f}}$, the current observable (31) can then be rewritten as

$$
\mathfrak{K}_{\mathcal{Z}, \mathcal{Z}^{(\tau)}}^{(\omega, \mathcal{E})}=\left\langle\mathrm{A}, K_{\mathcal{Z}, \mathcal{Z}^{(\tau)}}^{(\omega, \mathcal{E})} \mathrm{A}\right\rangle=\sum_{x, y \in \mathbb{Z}^{d}}\left\langle\mathfrak{e}_{x}, K_{\mathcal{Z}, \mathcal{Z}^{(\tau)}}^{(\omega, \mathcal{E}} \mathfrak{e}_{y}\right\rangle_{\mathfrak{h}} a\left(\mathfrak{e}_{x}\right)^{*} a\left(\mathfrak{e}_{y}\right)
$$

where $K_{\mathcal{Z}, \mathcal{Z}(\tau)}^{(\omega, \mathcal{E})} \in \mathcal{B}(\mathfrak{h})$ is the operator acting on the one-particle Hilbert space $\mathfrak{h}$ defined by

$$
\begin{aligned}
& K_{\mathcal{Z}, \mathcal{Z}(\tau)}^{(\omega, \mathcal{E})} \doteq 4 \sum_{k, q=1}^{d} w_{k} \sum_{Z \in \mathcal{Z}} \sum_{x, y, x+e_{k}, y+e_{q} \in Z} \int_{-\infty}^{0}\{\mathcal{E}(\alpha)\}_{q} \mathrm{~d} \alpha \\
& \int_{0}^{-\alpha} \mathrm{d} s i\left[\mathrm{e}^{-i s h_{\mathcal{Z}(\tau)}^{(\omega)}} \Im \mathrm{m}\left\{S_{y+e_{q}, y}^{(\omega)}\right\} \mathrm{e}^{i s h_{\mathcal{Z}(\tau)}^{(\omega)}}, \Im \mathrm{m}\left\{S_{x+e_{k}, x}^{(\omega)}\right\}\right] \\
& +2 \sum_{k=1}^{d} w_{k} \sum_{Z \in \mathcal{Z}} \sum_{x, x+e_{k} \in Z}\left(\int_{-\infty}^{0}\{\mathcal{E}(\alpha)\}_{q} \mathrm{~d} \alpha\right) \Re \mathrm{e}\left\{S_{x+e_{k}, x}^{(\omega)}\right\} .
\end{aligned}
$$

Note that the range of this bounded and self-adjoint operator is finite-dimensional whenever $\mathcal{Z} \in \mathfrak{Z}_{\mathrm{f}}$. 


\subsection{Differentiability Class of Generating Functions}

The aim of this section is to prove Theorem 3.1 (i), in particular that the generating function $s \mapsto \mathrm{J}^{(s \mathcal{E})}$ defined by 16 belongs to $C^{2}(\mathbb{R} ; \mathbb{R})$. By [1, Theorem 3.1], we already know that it is a well-defined, continuously differentiable, convex function. So, one has to prove here that the second derivative of the generating function exists and is continuous. To arrive at this assertion, we follow the lines of arguments of [1, Section 4] showing [1, Theorem 3.1] via the control of the thermodynamic limit of finite-volume generating functions that are random.

Fix once and for all $\beta \in \mathbb{R}^{+}, \lambda, \vartheta \in \mathbb{R}_{0}^{+}$and $\vec{w} \in \mathbb{R}^{d}$ with $\|\vec{w}\|_{\mathbb{R}^{d}}=1$. For any $\mathcal{E} \in C_{0}^{0}\left(\mathbb{R} ; \mathbb{R}^{d}\right)$, $\omega \in \Omega$ and three finite collections $\mathcal{Z}, \mathcal{Z}^{(\varrho)}, \mathcal{Z}^{(\tau)} \in \mathfrak{Z}_{\mathrm{f}}$, we define the finite-volume generating function

$$
\mathrm{J}_{\mathcal{Z}, \mathcal{Z}(\varrho), \mathcal{Z}(\tau)}^{(\omega, \mathcal{E}} \doteq g_{\mathcal{Z}, \mathcal{Z}(\varrho), \mathcal{Z}(\tau)}^{(\omega, \mathcal{E})}-g_{\mathcal{Z}, \mathcal{Z}(\varrho), \mathcal{Z}(\tau)}^{(\omega,)}
$$

where

$$
g_{\mathcal{Z}, \mathcal{Z}^{(\varrho), \mathcal{Z}(\tau)}}^{(\omega, \mathcal{E})} \doteq \frac{1}{|\cup \mathcal{Z}|} \ln \operatorname{tr}\left(\exp \left(-\beta\left\langle\mathrm{A}, h_{\mathcal{Z}^{(\varrho)}}^{(\omega)} \mathrm{A}\right\rangle\right) \exp \left(\mathfrak{K}_{\mathcal{Z}, \mathcal{Z}(\tau)}^{(\omega, \mathcal{E})}\right)\right)
$$

Recall that the tracial state $\operatorname{tr} \in \mathcal{U}^{*}$ is the gauge-invariant quasi-free state with two-point correlation function given by $\sqrt{6}$ for $\beta=0$, while $h_{\mathcal{Z}(e)}^{(\omega)}$ is the one-particle Hamiltonian defined by (29). See also (30) and (31). Compare (37)- (38) with the equalities

$$
\begin{aligned}
\mathrm{J}^{(\mathcal{E})} & \doteq \lim _{L \rightarrow \infty} \frac{1}{\left|\Lambda_{L}\right|} \mathbb{E}\left[\ln \varrho^{(\cdot)}\left(\mathrm{e}^{\left|\Lambda_{L}\right| \mathbb{I}_{\Lambda_{L}}^{(\cdot, \mathcal{E})}}\right)\right] \\
& =\lim _{L \rightarrow \infty} \frac{1}{\left|\Lambda_{L}\right|} \ln \varrho^{(\omega)}\left(\mathrm{e}^{\left|\Lambda_{L}\right| \mathbb{I}_{\Lambda_{L}}^{(\omega, \mathcal{E})}}\right)=\lim _{L \rightarrow \infty} \lim _{L_{\varrho} \rightarrow \infty} \lim _{L_{\tau} \rightarrow \infty} \mathrm{J}_{\left\{\Lambda_{L}\right\},\left\{\Lambda_{L_{\varrho}}\right\},\left\{\Lambda_{L \tau}\right\}},
\end{aligned}
$$

where the random variable $\omega$ is in a measurable subset of full measure ${ }^{10}$, by [1, Theorem 3.1 and Equation (45)]. Recall that $\Lambda_{\ell} \doteq\{\mathbb{Z} \cap[-\ell, \ell]\}^{d}$ for $\ell \in \mathbb{R}_{0}^{+}$. (See again (16) for the definition of the generating function.) In fact, by [1, Proposition 4.10], the above local generating functions can be approximately decomposed into boxes of fixed volume and we use the Ackoglu-Krengel (superadditive) ergodic theorem [1, Theorem 4.17] to deduce, via [1, Proposition 4.8], the existence of the generating functions as the thermodynamic limit of finite-volume generating functions, as given in (39).

In order to prove that the generating function is continuously differentiable, one uses in [1, Corollary 4.20] the (Arzelà-) Ascoli theorem [19, Theorem A5]. This approach requires uniform bounds on the first and second derivatives of the finite-volume generating functions

$$
s \mapsto \mathrm{J}_{\mathcal{Z}, \mathcal{Z}(\varrho), \mathcal{Z}(\tau)}^{(\omega, \mathcal{E})}, \quad \mathcal{E} \in C_{0}^{0}\left(\mathbb{R} ; \mathbb{R}^{d}\right), \omega \in \Omega, \mathcal{Z}, \mathcal{Z}^{(\varrho)}, \mathcal{Z}^{(\tau)} \in \mathfrak{Z}_{\mathrm{f}}
$$

This is done in [1, Proposition 4.9], which establishes the following: Fixing $\mathcal{E} \in C_{0}^{0}\left(\mathbb{R} ; \mathbb{R}^{d}\right)$ and $\beta_{1}, s_{1}, \vartheta_{1}, \lambda_{1} \in \mathbb{R}^{+}$, one has

$$
\sup _{\substack{\beta \in\left(0, \beta_{1}\right], \vartheta \in\left[0, \vartheta_{1}\right], \lambda \in\left[0, \lambda_{1}\right] \\ \omega \in \Omega, s \in\left[-s_{1}, s_{1}\right], \mathcal{Z}, \mathcal{Z}^{(\varrho)}, \mathcal{Z}^{(\tau)} \in \mathcal{Z}_{\mathrm{f}}}}\left\{\left|\partial_{s} \mathrm{~J}_{\mathcal{Z}, \mathcal{Z}(\varrho), \mathcal{Z}(\tau)}^{(\omega, s \mathcal{E})}\right|+\left|\partial_{s}^{2} \mathrm{~J}_{\mathcal{Z}, \mathcal{Z}(\varrho), \mathcal{Z}(\tau)}^{(\omega, s \mathcal{E})}\right|\right\}<\infty .
$$

In order to get in the same way the existence and continuity of the second derivative of the generating function, we need now to control the third-order derivative of the same finite-volume generating functions 40 .

\footnotetext{
${ }^{10}$ The measurable subset $\tilde{\Omega} \subseteq \Omega$ of full measure of [1, Theorem 3.1] does not depend on $\beta \in \mathbb{R}^{+}, \vartheta, \lambda \in \mathbb{R}_{0}^{+}$, $\mathcal{E} \in C_{0}^{0}\left(\mathbb{R} ; \mathbb{R}^{d}\right)$ and $\vec{w} \in \mathbb{R}^{d}$ with $\|\vec{w}\|_{\mathbb{R}^{d}}=1$.
} 
Equation (41) is proven by using the CAR (4) and the Combes-Thomas estimate [1, Appendix A], in particular the bound

$$
\sup _{\lambda \in \mathbb{R}_{0}^{+}} \sup _{\mathcal{Z} \in \mathfrak{Z}} \sup _{\omega \in \Omega}\left|\left\langle\mathfrak{e}_{x}, \mathrm{e}^{i t h_{\mathcal{Z}}^{(\omega)}} \mathfrak{e}_{y}\right\rangle_{\mathfrak{h}}\right| \leq 36 \mathrm{e}^{|t \eta|-2 \mu_{\eta}|x-y|}, \quad x, y \in \mathbb{Z}^{d}, \vartheta \in \mathbb{R}_{0}^{+}, t \in \mathbb{R},
$$

(see [1, Equation (7)]), where

$$
\mu_{\eta} \doteq \mu \min \left\{\frac{1}{2}, \frac{\eta}{8 d(1+\vartheta) \mathrm{e}^{\mu}}\right\}
$$

the parameters $\eta, \mu \in \mathbb{R}^{+}$being two arbitrarily fixed (strictly positive) constants. For any $\mathcal{E} \in C_{0}^{0}\left(\mathbb{R} ; \mathbb{R}^{d}\right)$ and $\beta_{1}, s_{1}, \vartheta_{1}, \lambda_{1} \in \mathbb{R}^{+}$, the Combes-Thomas estimate leads also to the uniform estimates

$$
\sup _{\substack{\beta \in\left(0, \beta_{1}\right], \vartheta \in\left[0, \vartheta_{1}\right], \lambda \in\left[0, \lambda_{1}\right] \\ \omega \in \Omega, s \in\left[-s_{1}, s_{1}\right], \mathcal{Z}, \mathcal{Z}^{(e)}, \mathcal{Z}^{(\tau)} \in \mathfrak{J}_{\mathrm{f}}}} \sup _{x \in \mathbb{Z}^{d}} \sum_{y \in \mathbb{Z}^{d}}\left|\left\langle\mathfrak{e}_{y}, \frac{1}{1+\mathrm{e}^{-\frac{s}{2} K_{\mathcal{Z}, \mathcal{Z}(\tau)}^{(\omega, \mathcal{E})} \mathrm{e}^{\beta h_{\mathcal{Z}(\varrho)}^{(\omega)}} \mathrm{e}^{-\frac{s}{2} K_{\mathcal{Z}, \mathcal{Z}(\tau)}^{(\omega, \mathcal{E})}}} \mathfrak{e}_{x}}\right\rangle\right|<\infty
$$

(see the end of the proof of [1, Proposition 4.9]) as well as

$$
\sup _{\vartheta \in\left[0, \vartheta_{1}\right]} \sup _{\lambda \in \mathbb{R}_{0}^{+}} \sup _{\mathcal{Z}, \mathcal{Z}^{(\tau)} \in \mathfrak{Z}_{\mathfrak{f}}} \sup _{\omega \in \Omega} \frac{1}{|\cup \mathcal{Z}|} \sum_{x, y \in \mathbb{Z}^{d}}\left|\left\langle\mathfrak{e}_{x}, K_{\mathcal{Z}, \mathcal{Z}^{(\tau)}}^{(\omega, \mathcal{E})} \mathfrak{e}_{y}\right\rangle_{\mathfrak{h}}\right|<\infty
$$

and

$$
\sup _{\vartheta \in\left[0, \vartheta_{1}\right]} \sup _{\lambda \in \mathbb{R}_{0}^{+}} \sup _{\mathcal{Z}, \mathcal{Z}(\tau) \in \mathcal{Z}_{\mathfrak{f}}} \sup _{\omega \in \Omega}\left|\left\langle\mathfrak{e}_{x}, K_{\mathcal{Z}, \mathcal{Z}^{(\tau)}}^{(\omega, \mathcal{E})} \mathfrak{e}_{y}\right\rangle_{\mathfrak{h}}\right| \leq C_{x, y}^{\left(\mathcal{E}, \vartheta_{1}\right)}<\infty
$$

for $x, y \in \mathbb{Z}^{d}$, where $C_{x, y}^{\left(\mathcal{E}, \vartheta_{1}\right)} \in \mathbb{R}^{+}$are constants satisfying

$$
\sup _{x, y \in \mathbb{Z}^{d}} C_{x, y}^{\left(\mathcal{E}, \vartheta_{1}\right)}<\infty \quad \text { and } \quad \sup _{x \in \mathbb{Z}^{d}} \sum_{y \in \mathbb{Z}^{d}} C_{x, y}^{\left(\mathcal{E}, \vartheta_{1}\right)}<\infty .
$$

Recall that $K_{\mathcal{Z}, \mathcal{Z}^{(\tau)}}^{(\omega, \mathcal{E})} \in \mathcal{B}(\mathfrak{h})$ is the operator defining linear response current observables, by (35)-(36).

In order to give a uniform estimate on the third-order derivative of the finite-volume generating functions (40), similar to the proof of Equation (41), we use again the Combes-Thomas estimate, which yields (44)-(47). This proof bears however on more complex computations than the one of Equation (41), which only controls the first and second derivatives of the same function.

\section{Proposition 4.1 (Uniform boundedness of third derivatives)}

Fix an electric field $\mathcal{E} \in C_{0}^{0}\left(\mathbb{R} ; \mathbb{R}^{d}\right), \vec{w} \in \mathbb{R}^{d}$ with $\|\vec{w}\|_{\mathbb{R}^{d}}=1$ and the parameters $\beta_{1}, s_{1}, \vartheta_{1}, \lambda_{1} \in$ $\mathbb{R}^{+}$. Then,

$$
\sup _{\substack{\beta \in\left(0, \beta_{1}\right], \vartheta \in\left[0, \vartheta_{1}\right], \quad \lambda \in\left[0, \lambda_{1}\right] \\ \omega \in \Omega, s \in\left[-s_{1}, s_{1}\right], \mathcal{Z}, \mathcal{Z}^{(\varrho)}, \mathcal{Z}^{(\tau)} \in \mathfrak{Z}_{\mathrm{f}}}}\left|\partial_{s}^{3} \mathrm{~J}_{\mathcal{Z}, \mathcal{Z}(\varrho), \mathcal{Z}(\tau)}^{(\omega, \mathcal{E})}\right|<\infty .
$$

Proof. For any $\beta \in \mathbb{R}^{+}, \vartheta, \lambda \in \mathbb{R}_{0}^{+}, \mathcal{E} \in C_{0}^{0}\left(\mathbb{R} ; \mathbb{R}^{d}\right), \vec{w} \in \mathbb{R}^{d}$ with $\|\vec{w}\|_{\mathbb{R}^{d}}=1$ and $\mathcal{Z}, \mathcal{Z}^{(\varrho)}, \mathcal{Z}^{(\tau)} \in$ $\mathfrak{Z}_{\mathrm{f}}$, a straightforward computation yields that

$$
\begin{aligned}
& \partial_{s}^{3} \mathrm{~J}_{\mathcal{Z}, \mathcal{Z}(\varrho), \mathcal{Z}(\tau)}^{(\omega, \mathcal{E})} \\
= & \frac{1}{|\cup \mathcal{Z}|} \varpi_{s}^{T}\left(\mathfrak{K}_{\mathcal{Z}, \mathcal{Z}^{(\tau)}}^{(\omega, \mathcal{E})} ; \mathfrak{K}_{\mathcal{Z}, \mathcal{Z}(\tau)}^{(\omega, \mathcal{E})} ; \mathfrak{K}_{\mathcal{Z}, \mathcal{Z}(\tau)}^{(\omega, \mathcal{E})}\right) \\
= & \frac{1}{|\cup \mathcal{Z}|}\left(\varpi_{s}\left(\left(\mathfrak{K}_{\mathcal{Z}, \mathcal{Z}(\tau)}^{(\omega, \mathcal{E})}\right)^{3}\right)-3 \varpi_{s}\left(\left(\mathfrak{K}_{\mathcal{Z}, \mathcal{Z}(\tau)}^{(\omega, \mathcal{E})}\right)^{2}\right) \varpi_{s}\left(\mathfrak{K}_{\mathcal{Z}, \mathcal{Z}^{(\tau)}}^{(\omega, \mathcal{E})}\right)+2 \varpi_{s}\left(\mathfrak{K}_{\mathcal{Z}, \mathcal{Z}^{(\tau)}}^{(\omega, \mathcal{E})}\right)^{3}\right),
\end{aligned}
$$


where $\varpi_{s}$ is the (unique) gauge-invariant quasi-free state satisfying

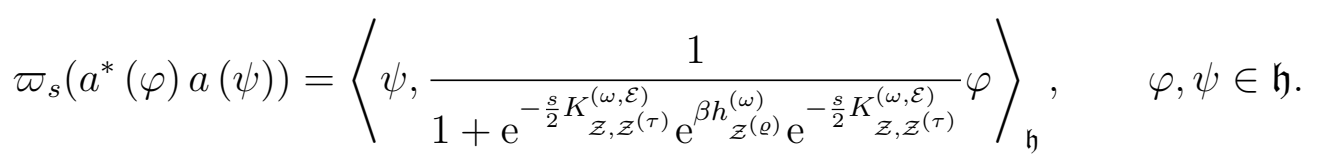

In the first equality of $(48), \varpi_{s}^{T}(\cdot ; \cdot ; \cdot)$ denotes the so-called "truncated" or "connected" correlation function of third order, associated with the state $\varpi_{s}$. Recall that, for all $A_{1}, A_{2}, A_{3} \in \mathcal{U}$, this function is defined by

$$
\begin{aligned}
\varpi_{s}^{T}\left(A_{1} ; A_{2} ; A_{3}\right) \doteq & \varpi_{s}\left(A_{1} A_{2} A_{3}\right)-\varpi_{s}\left(A_{1}\right) \varpi_{s}\left(A_{2} A_{3}\right)-\varpi_{s}\left(A_{2}\right) \varpi_{s}\left(A_{1} A_{3}\right) \\
& -\varpi_{s}\left(A_{3}\right) \varpi_{s}\left(A_{1} A_{2}\right)+2 \varpi_{s}\left(A_{1}\right) \varpi_{s}\left(A_{2}\right) \varpi_{s}\left(A_{3}\right) .
\end{aligned}
$$

(This is similar to [1, Proof of Proposition 4.9, until Equation (48)].) Recall that $\left\{\mathfrak{e}_{x}\right\}_{x \in \mathbb{Z}^{d}}$ is the canonical orthonormal basis of $\mathfrak{h}$, which is defined by $\mathfrak{e}_{x}(y) \doteq \delta_{x, y}$ for all $x, y \in \mathbb{Z}^{d}$. By linearity and continuity in each argument of $\varpi_{s}^{T}(\cdot ; \cdot ; \cdot)$, one has

$$
\begin{aligned}
\partial_{s}^{3} J_{\mathcal{Z}, \mathcal{Z}(e), \mathcal{Z}(\tau)}^{(\omega, s \mathcal{E})}= & \frac{1}{|\cup \mathcal{Z}|} \sum_{x_{1}, y_{1}, x_{2}, y_{2}, x_{3}, y_{3} \in \mathbb{Z}^{d}}\left\langle\mathfrak{e}_{x_{1}}, K_{\mathcal{Z}, \mathcal{Z}(\tau)}^{(\omega, \mathcal{E})} \mathfrak{e}_{y_{1}}\right\rangle_{\mathfrak{h}}\left\langle\mathfrak{e}_{x_{2}}, K_{\mathcal{Z}, \mathcal{Z}(\tau)}^{(\omega, \mathcal{E})} \mathfrak{e}_{y_{2}}\right\rangle_{\mathfrak{h}}\left\langle\mathfrak{e}_{x_{3}}, K_{\mathcal{Z}, \mathcal{Z}(\tau)}^{(\omega, \mathcal{E})} \mathfrak{e}_{y_{3}}\right\rangle_{\mathfrak{h}} \\
& \times \varpi_{s}^{T}\left(a^{*}\left(\mathfrak{e}_{x_{1}}\right) a\left(\mathfrak{e}_{y_{1}}\right) ; a^{*}\left(\mathfrak{e}_{x_{2}}\right) a\left(\mathfrak{e}_{y_{2}}\right) ; a^{*}\left(\mathfrak{e}_{x_{3}}\right) a\left(\mathfrak{e}_{y_{3}}\right)\right) .
\end{aligned}
$$

Note that, by Equation (8) and the fact that $\varpi_{s}$ is a gauge-invariant quasi-free state,

$$
\begin{aligned}
& \varpi_{s}\left(a^{*}\left(\mathfrak{e}_{x_{1}}\right) a\left(\mathfrak{e}_{y_{1}}\right) a^{*}\left(\mathfrak{e}_{x_{2}}\right) a\left(\mathfrak{e}_{y_{2}}\right) a^{*}\left(\mathfrak{e}_{x_{3}}\right) a\left(\mathfrak{e}_{y_{3}}\right)\right) \\
= & \operatorname{det}\left(\begin{array}{ccc}
\varpi_{s}\left(a^{*}\left(\mathfrak{e}_{x_{1}}\right) a\left(\mathfrak{e}_{y_{1}}\right)\right) & \varpi_{s}\left(a^{*}\left(\mathfrak{e}_{x_{1}}\right) a\left(\mathfrak{e}_{y_{2}}\right)\right) & \varpi_{s}\left(a^{*}\left(\mathfrak{e}_{x_{1}}\right) a\left(\mathfrak{e}_{y_{3}}\right)\right) \\
-\varpi_{s}\left(a\left(\mathfrak{e}_{y_{1}}\right) a^{*}\left(\mathfrak{e}_{x_{2}}\right)\right) & \varpi_{s}\left(a^{*}\left(\mathfrak{e}_{x_{2}}\right) a\left(\mathfrak{e}_{y_{2}}\right)\right) & \varpi_{s}\left(a^{*}\left(\mathfrak{e}_{x_{2}}\right) a\left(\mathfrak{e}_{y_{3}}\right)\right) \\
-\varpi_{s}\left(a\left(\mathfrak{e}_{y_{1}}\right) a^{*}\left(\mathfrak{e}_{x_{3}}\right)\right) & -\varpi_{s}\left(a\left(\mathfrak{e}_{y_{2}}\right) a^{*}\left(\mathfrak{e}_{x_{3}}\right)\right) & \varpi_{s}\left(a^{*}\left(\mathfrak{e}_{x_{3}}\right) a\left(\mathfrak{e}_{y_{3}}\right)\right)
\end{array}\right) \\
= & \sum_{g \in \mathcal{G}_{3}} \xi_{s}^{g}\left(x_{1}, y_{1}, x_{2}, y_{2}, x_{3}, y_{3}\right)
\end{aligned}
$$

(use, for instance, [37, Lemma 3.1] to get the above determinant), where

$$
\begin{aligned}
\mathcal{G}_{3} \doteq & \{\{(1,1),(2,2),(3,3)\},\{(1,1),(2,3),(3,2)\},\{(1,2),(2,1),(3,3)\}\} \\
& \cup\{\{(1,2),(2,3),(3,1)\},\{(1,3),(2,1),(3,2)\},\{(1,3),(2,2),(3,1)\}\}
\end{aligned}
$$

is a set of oriented graphs with vertex set $\{1,2,3\}$ and

$$
\begin{aligned}
& \xi_{s}^{\{(1,1),(2,2),(3,3)\}}\left(x_{1}, y_{1}, x_{2}, y_{2}, x_{3}, y_{3}\right) \doteq \varpi_{s}\left(a^{*}\left(\mathfrak{e}_{x_{1}}\right) a\left(\mathfrak{e}_{y_{1}}\right)\right) \varpi_{s}\left(a^{*}\left(\mathfrak{e}_{x_{2}}\right) a\left(\mathfrak{e}_{y_{2}}\right)\right) \varpi_{s}\left(a^{*}\left(\mathfrak{e}_{x_{3}}\right) a\left(\mathfrak{e}_{y_{3}}\right)\right), \\
& \xi_{s}^{\{(1,1),(2,3),(3,2)\}}\left(x_{1}, y_{1}, x_{2}, y_{2}, x_{3}, y_{3}\right) \doteq \varpi_{s}\left(a^{*}\left(\mathfrak{e}_{x_{1}}\right) a\left(\mathfrak{e}_{y_{1}}\right)\right) \varpi_{s}\left(a^{*}\left(\mathfrak{e}_{x_{2}}\right) a\left(\mathfrak{e}_{y_{3}}\right)\right) \varpi_{s}\left(a\left(\mathfrak{e}_{y_{2}}\right) a^{*}\left(\mathfrak{e}_{x_{3}}\right)\right), \\
& \xi_{s}^{\{(1,2),(2,1),(3,3)\}}\left(x_{1}, y_{1}, x_{2}, y_{2}, x_{3}, y_{3}\right) \doteq \varpi_{s}\left(a^{*}\left(\mathfrak{e}_{x_{1}}\right) a\left(\mathfrak{e}_{y_{2}}\right)\right) \varpi_{s}\left(a\left(\mathfrak{e}_{y_{1}}\right) a^{*}\left(\mathfrak{e}_{x_{2}}\right)\right) \varpi_{s}\left(a^{*}\left(\mathfrak{e}_{x_{3}}\right) a\left(\mathfrak{e}_{y_{3}}\right)\right), \\
& \xi_{s}^{\{(1,2),(2,3),(3,1)\}}\left(x_{1}, y_{1}, x_{2}, y_{2}, x_{3}, y_{3}\right) \doteq-\varpi_{s}\left(a^{*}\left(\mathfrak{e}_{x_{1}}\right) a\left(\mathfrak{e}_{y_{2}}\right)\right) \varpi_{s}\left(a^{*}\left(\mathfrak{e}_{x_{2}}\right) a\left(\mathfrak{e}_{y_{3}}\right)\right) \varpi_{s}\left(a\left(\mathfrak{e}_{y_{1}}\right) a^{*}\left(\mathfrak{e}_{x_{3}}\right)\right), \\
& \xi_{s}^{\{(1,3),(2,1),(3,2)\}}\left(x_{1}, y_{1}, x_{2}, y_{2}, x_{3}, y_{3}\right) \doteq \varpi_{s}\left(a^{*}\left(\mathfrak{e}_{x_{1}}\right) a\left(\mathfrak{e}_{y_{3}}\right)\right) \varpi_{s}\left(a\left(\mathfrak{e}_{y_{1}}\right) a^{*}\left(\mathfrak{e}_{x_{2}}\right)\right) \varpi_{s}\left(a\left(\mathfrak{e}_{y_{2}}\right) a^{*}\left(\mathfrak{e}_{x_{3}}\right)\right), \\
& \xi_{s}^{\{(1,3),(2,2),(3,1)\}}\left(x_{1}, y_{1}, x_{2}, y_{2}, x_{3}, y_{3}\right) \doteq \varpi_{s}\left(a^{*}\left(\mathfrak{e}_{x_{1}}\right) a\left(\mathfrak{e}_{y_{3}}\right)\right) \varpi_{s}\left(a^{*}\left(\mathfrak{e}_{x_{2}}\right) a\left(\mathfrak{e}_{y_{2}}\right)\right) \varpi_{s}\left(a\left(\mathfrak{e}_{y_{1}}\right) a^{*}\left(\mathfrak{e}_{x_{3}}\right)\right) .
\end{aligned}
$$

By elementary computations, one sees that taking connected correlations corresponds here, as is usual, to only keep the terms associated with connected graphs. That is,

$$
\begin{aligned}
\varpi_{s}^{T}\left(a^{*}\left(\mathfrak{e}_{x_{1}}\right) a\left(\mathfrak{e}_{y_{1}}\right)\right. & ; a^{*}\left(\mathfrak{e}_{x_{2}}\right) a\left(\mathfrak{e}_{y_{2}}\right) \\
\left.=\varpi_{s}\left(a^{*}\left(\mathfrak{e}_{x_{1}}\right) a\left(\mathfrak{e}_{x_{3}}\right)\right) a\left(\mathfrak{e}_{y_{3}}\right)\right) & \varpi_{s}\left(a\left(\mathfrak{e}_{y_{1}}\right) a^{*}\left(\mathfrak{e}_{x_{2}}\right)\right) \varpi_{s}\left(a\left(\mathfrak{e}_{y_{2}}\right) a^{*}\left(\mathfrak{e}_{x_{3}}\right)\right) \\
& \quad-\varpi_{s}\left(a^{*}\left(\mathfrak{e}_{x_{1}}\right) a\left(\mathfrak{e}_{y_{2}}\right)\right) \varpi_{s}\left(a^{*}\left(\mathfrak{e}_{x_{2}}\right) a\left(\mathfrak{e}_{y_{3}}\right)\right) \varpi_{s}\left(a\left(\mathfrak{e}_{y_{1}}\right) a^{*}\left(\mathfrak{e}_{x_{3}}\right)\right) .
\end{aligned}
$$


Hence,

$$
\partial_{s}^{3} \mathrm{~J}_{\mathcal{Z}, \mathcal{Z}(\varrho), \mathcal{Z}(\tau)}^{(\omega, \mathcal{E})}=\mathbf{K}_{1}-\mathbf{K}_{2}
$$

where

$$
\begin{array}{r}
\mathbf{K}_{1} \doteq \frac{1}{|\cup \mathcal{Z}|} \sum_{x_{1}, y_{1}, x_{2}, y_{2}, x_{3}, y_{3} \in \mathbb{Z}^{d}}\left\langle\mathfrak{e}_{x_{1}}, K_{\mathcal{Z}, \mathcal{Z}^{(\tau)}}^{(\omega, \mathcal{E})} \mathfrak{e}_{y_{1}}\right\rangle_{\mathfrak{h}}\left\langle\mathfrak{e}_{x_{2}}, K_{\mathcal{Z}, \mathcal{Z}(\tau)}^{(\omega, \mathcal{E})} \mathfrak{e}_{y_{2}}\right\rangle_{\mathfrak{h}}\left\langle\mathfrak{e}_{x_{3}}, K_{\left.\mathcal{Z}, \mathcal{Z}^{(\tau)} \mathfrak{e}_{y_{3}}\right\rangle_{\mathfrak{h}}}\right. \\
\varpi_{s}\left(a^{*}\left(\mathfrak{e}_{x_{1}}\right) a\left(\mathfrak{e}_{y_{3}}\right)\right) \varpi_{s}\left(a\left(\mathfrak{e}_{y_{1}}\right) a^{*}\left(\mathfrak{e}_{x_{2}}\right)\right) \varpi_{s}\left(a\left(\mathfrak{e}_{y_{2}}\right) a^{*}\left(\mathfrak{e}_{x_{3}}\right)\right)
\end{array}
$$

and

$$
\begin{gathered}
\mathbf{K}_{2} \doteq \frac{1}{|\cup \mathcal{Z}|} \sum_{x_{1}, y_{1}, x_{2}, y_{2}, x_{3}, y_{3} \in \mathbb{Z}^{d}}\left\langle\mathfrak{e}_{x_{1}}, K_{\mathcal{Z}, \mathcal{Z}^{(\tau)}}^{(\omega, \mathcal{E})} \mathfrak{e}_{y_{1}}\right\rangle_{\mathfrak{h}}\left\langle\mathfrak{e}_{x_{2}}, K_{\mathcal{Z}, \mathcal{Z}^{(\tau)}}^{(\omega, \mathcal{E})} \mathfrak{e}_{y_{2}}\right\rangle_{\mathfrak{h}}\left\langle\mathfrak{e}_{x_{3}}, K_{\mathcal{Z}, \mathcal{Z}^{(\tau)}}^{(\omega, \mathcal{E})} \mathfrak{e}_{y_{3}}\right\rangle_{\mathfrak{h}} \\
\varpi_{s}\left(a^{*}\left(\mathfrak{e}_{x_{1}}\right) a\left(\mathfrak{e}_{y_{2}}\right)\right) \varpi_{s}\left(a\left(\mathfrak{e}_{y_{1}}\right) a^{*}\left(\mathfrak{e}_{x_{3}}\right)\right) \varpi_{s}\left(a^{*}\left(\mathfrak{e}_{x_{2}}\right) a\left(\mathfrak{e}_{y_{3}}\right)\right) .
\end{gathered}
$$

Applying the triangle inequality, we now obtain that

$$
\begin{aligned}
& \left|\mathbf{K}_{1}\right| \leq \frac{1}{|\cup \mathcal{Z}|} \sum_{x_{1}, y_{1}, x_{2}, y_{2}, x_{3}, y_{3} \in \mathbb{Z}^{d}}\left|\left\langle\mathfrak{e}_{x_{1}}, K_{\mathcal{Z}, \mathcal{Z}^{(\tau)}}^{(\omega, \mathcal{E})} \mathfrak{e}_{y_{1}}\right\rangle_{\mathfrak{h}}\right|\left|\left\langle\mathfrak{e}_{x_{2}}, K_{\mathcal{Z}, \mathcal{Z}^{(\tau)}}^{(\omega, \mathcal{E})} \mathfrak{e}_{y_{2}}\right\rangle_{\mathfrak{h}}\right|\left|\left\langle\mathfrak{e}_{x_{3}}, K_{\mathcal{Z}, \mathcal{Z}^{(\tau)}}^{(\omega, \mathcal{E})} \mathfrak{e}_{y_{3}}\right\rangle_{\mathfrak{h}}\right| \\
& \left|\varpi_{s}\left(a^{*}\left(\mathfrak{e}_{x_{1}}\right) a\left(\mathfrak{e}_{y_{3}}\right)\right)\right|\left|\varpi_{s}\left(a\left(\mathfrak{e}_{y_{1}}\right) a^{*}\left(\mathfrak{e}_{x_{2}}\right)\right)\right|\left|\varpi_{s}\left(a\left(\mathfrak{e}_{y_{2}}\right) a^{*}\left(\mathfrak{e}_{x_{3}}\right)\right)\right| \\
& \leq \sup _{x_{3}, y_{3} \in \mathbb{Z}^{d}}\left|\left\langle\mathfrak{e}_{x_{3}}, K_{\mathcal{Z}, \mathcal{Z}(\tau)}^{(\omega, \mathcal{E})} \mathfrak{e}_{y_{3}}\right\rangle_{\mathfrak{h}}\right| \sup _{x_{2} \in \mathbb{Z}^{d}} \sum_{y_{2} \in \mathbb{Z}^{d}}\left|\left\langle\mathfrak{e}_{x_{2}}, K_{\mathcal{Z}, \mathcal{Z}(\tau)}^{(\omega, \mathcal{E})} \mathfrak{e}_{y_{2}}\right\rangle_{\mathfrak{h}}\right| \frac{1}{|\cup \mathcal{Z}|} \sum_{x_{1}, y_{1} \in \mathbb{Z}^{d}}\left|\left\langle\mathfrak{e}_{x_{1}}, K_{\mathcal{Z}, \mathcal{Z}^{(\tau)}}^{(\omega, \mathcal{E})} \mathfrak{e}_{y_{1}}\right\rangle_{\mathfrak{h}}\right| \\
& \sup _{x_{1} \in \mathbb{Z}^{d}} \sum_{y_{3} \in \mathbb{Z}^{d}}\left|\varpi_{s}\left(a^{*}\left(\mathfrak{e}_{x_{1}}\right) a\left(\mathfrak{e}_{y_{3}}\right)\right)\right| \sup _{y_{1} \in \mathbb{Z}^{d}} \sum_{x_{2} \in \mathbb{Z}^{d}}\left|\varpi_{s}\left(a\left(\mathfrak{e}_{y_{1}}\right) a^{*}\left(\mathfrak{e}_{x_{2}}\right)\right)\right| \sup _{y_{2} \in \mathbb{Z}^{d}} \sum_{x_{3} \in \mathbb{Z}^{d}}\left|\varpi_{s}\left(a\left(\mathfrak{e}_{y_{2}}\right) a^{*}\left(\mathfrak{e}_{x_{3}}\right)\right)\right| .
\end{aligned}
$$

We can finally use Equations (44)-(47) and (49) to arrive from the last upper bound at

$$
\sup _{\substack{\beta \in\left(0, \beta_{1}\right], \vartheta \in\left[0, \vartheta_{1}\right], \lambda \in\left[0, \lambda_{1}\right] \\ \omega \in \Omega, s \in\left[-s_{1}, s_{1}\right], \mathcal{Z}, \mathcal{Z}^{(\varrho)}, \mathcal{Z}^{(\tau)} \in \mathfrak{Z}_{\mathrm{f}}}}\left|\mathbf{K}_{1}\right|<\infty .
$$

The absolute value $\left|\mathbf{K}_{2}\right|$ of the other term of $\partial_{s}^{3} J_{\mathcal{Z}, \mathcal{Z}(\varrho), \mathcal{Z}(\tau)}^{(\omega, \mathcal{E})}($ see $(50)-(52)$ ) can be bounded exactly in the same way. By the triangle inequality applied to $(50)$, this concludes the proof.

We can now sharpen the result given in [1, Corollary 4.20], stating that the mapping $s \mapsto$ $\mathrm{J}^{(s \mathcal{E})}$ defined by $(16)$ is continuously differentiable with

$$
\partial_{s} \mathrm{~J}^{(s \mathcal{E})}=\lim _{L \rightarrow \infty} \frac{\varrho^{(\omega)}\left(\mathbb{I}_{\Lambda_{L}^{(\omega, \mathcal{E})}} \mathrm{e}^{s\left|\Lambda_{L}\right| \mathbb{I}_{L}^{(\omega, \mathcal{E})}}\right)}{\varrho^{(\omega)}\left(\mathrm{e}^{s\left|\Lambda_{L}\right| \mathbb{I}_{\Lambda_{L}}^{(\omega, \mathcal{E})}}\right)} .
$$

Thanks to Equation (41) and Proposition 4.1, we now obtain the following assertion:

\section{Corollary 4.2 (Differentiability of generating functions)}

There is a measurable subset $\tilde{\Omega} \subseteq \Omega$ of full measure such that, for all $\beta \in \mathbb{R}^{+}, \vartheta, \lambda \in \mathbb{R}_{0}^{+}$, $\omega \in \tilde{\Omega}, \mathcal{E} \in C_{0}^{0}\left(\mathbb{R} ; \mathbb{R}^{d}\right)$ and $\vec{w} \in \overline{\mathbb{R}}^{d}$ with $\|\vec{w}\|_{\mathbb{R}^{d}}=1$, the mapping $s \mapsto \mathrm{J}^{(s \mathcal{E})}$ from $\mathbb{R}$ to itself belongs to $C^{2}(\mathbb{R} ; \mathbb{R})$ and

$$
\begin{aligned}
&\left.\partial_{s} \mathrm{~J}^{(s \mathcal{E})}\right|_{s=0}=x^{(\mathcal{E})} \doteq \lim _{L \rightarrow \infty} \mathbb{E}\left[\varrho^{(\cdot)}\left(\mathbb{I}_{\Lambda_{L}^{(\cdot \mathcal{E})}}\right)\right]=\lim _{L \rightarrow \infty} \varrho^{(\omega)}\left(\mathbb{I}_{\Lambda_{L}}^{(\omega, \mathcal{E})}\right) \\
&\left.\partial_{s}^{2} \mathrm{~J}^{(s \mathcal{E})}\right|_{s=0}=\lim _{L \rightarrow \infty} \mathbb{E}\left[\mathbf{F}_{L}^{(\cdot, \mathcal{E})}\right]=\lim _{L \rightarrow \infty} \mathbf{F}_{L}^{(\omega, \mathcal{E})} \geq 0,
\end{aligned}
$$

where $\mathbf{F}_{L}^{(\omega, \mathcal{E})}$ is the quantum fluctuation of the linear response current defined by (18) for any $L \in \mathbb{R}_{0}^{+}$. See also (13) for the definition of the macroscopic current density $x^{(\mathcal{E})}$. 
Proof. [1, Corollary 4.19] states, among other things, the existence of a measurable set $\tilde{\Omega}$ of full measure such that, for all $\beta \in \mathbb{R}^{+}, \vartheta, \lambda \in \mathbb{R}_{0}^{+}, \omega \in \tilde{\Omega}, \mathcal{E} \in C_{0}^{0}\left(\mathbb{R} ; \mathbb{R}^{d}\right), \vec{w} \in \mathbb{R}^{d}$ with $\|\vec{w}\|_{\mathbb{R}^{d}}=1$ and $s \in \mathbb{R}$

$$
\mathrm{J}^{(s \mathcal{E})}=\lim _{L_{\tau} \geq L_{\varrho} \geq L \rightarrow \infty} \mathrm{J}_{\left\{\Lambda_{L}\right\},\left\{\Lambda_{L_{\varrho}}\right\},\left\{\Lambda_{L_{\tau}}\right\}}^{(\omega, s \mathcal{E}} .
$$

Fix from now all parameters $\beta \in \mathbb{R}^{+}, \vartheta, \lambda \in \mathbb{R}_{0}^{+}, \omega \in \tilde{\Omega}, \mathcal{E} \in C_{0}^{0}\left(\mathbb{R} ; \mathbb{R}^{d}\right)$ and $\vec{w} \in \mathbb{R}^{d}$ with $\|\vec{w}\|_{\mathbb{R}^{d}}=1$. By combining Equation (41) and Proposition 4.1 with the mean value theorem and the (Arzelà-) Ascoli theorem [19, Theorem A5], there are three sequences

$$
\left\{L_{\tau}^{(n)}\right\}_{n \in \mathbb{N}},\left\{L_{\varrho}^{(n)}\right\}_{n \in \mathbb{N}},\left\{L^{(n)}\right\}_{n \in \mathbb{N}} \subseteq \mathbb{R}_{0}^{+},
$$

with $L_{\tau}^{(n)} \geq L_{\varrho}^{(n)} \geq L^{(n)}$, such that, as $n \rightarrow \infty$, the mappings

$$
s \mapsto \mathrm{J}_{\left\{\Lambda_{L}^{(n)}\right\},\left\{\Lambda_{L_{\varrho}^{(n)}}^{(\omega, s \mathcal{E})},\left\{\Lambda_{L_{\tau}^{(n)}}\right\}\right.}, \quad s \mapsto \partial_{s} \mathrm{~J}_{\left\{\Lambda_{L^{(n)}}^{(\omega, s \mathcal{E})}\right\},\left\{\Lambda_{L_{\varrho}^{(n)}}\right\},\left\{\Lambda_{L_{\tau}^{(n)}}\right\}} \quad \text { and } \quad s \mapsto \partial_{s}^{2} \mathrm{~J}_{\left\{\Lambda_{L}^{(n)}\right\},\left\{\Lambda_{L_{\varrho}^{(n)}}^{(\omega, s \mathcal{E})},\left\{\Lambda_{L_{\tau}^{(n)}}\right\}\right.}
$$

from $\mathbb{R}$ to itself converge uniformly for $s$ in any compact subset of $\mathbb{R}$. So, the mapping $s \mapsto \mathrm{J}^{(s \mathcal{E})}$ from $\mathbb{R}$ to itself is a $C^{2}$-function with

$$
\partial_{s} \mathrm{~J}^{(s \mathcal{E})}=\lim _{L_{\tau} \geq L_{\varrho} \geq L \rightarrow \infty} \partial_{s} \mathrm{~J}_{\left\{\Lambda_{L}\right\},\left\{\Lambda_{L_{\varrho}}\right\},\left\{\Lambda_{\left.L_{\tau}\right\}}\right.}^{(\omega, \mathcal{E})}=\lim _{L \rightarrow \infty}\left(\frac{\varrho^{(\omega)}\left(\mathbb{I}_{\Lambda_{L}}^{(\omega, \mathcal{E})} \mathrm{e}^{s\left|\Lambda_{L}\right| \mathbb{I}_{\Lambda_{L}}^{(\omega, \mathcal{E})}}\right)}{\varrho^{(\omega)}\left(\mathrm{e}^{s\left|\Lambda_{L}\right| \mathbb{I}_{\Lambda_{L}}^{(\omega, \mathcal{E})}}\right)}\right)
$$

and

$$
\begin{aligned}
\partial_{s}^{2} \mathrm{~J}^{(s \mathcal{E})}= & \lim _{L_{\tau} \geq L_{\varrho} \geq L \rightarrow \infty} \partial_{s}^{2} \mathrm{~J}_{\left\{\Lambda_{L}\right\},\left\{\Lambda_{L_{\varrho}}\right\},\left\{\Lambda_{L \tau}\right\}}^{(\omega, \mathcal{E})} \\
= & \lim _{L \rightarrow \infty}\left|\Lambda_{L}\right|\left(\frac{\varrho^{(\omega)}\left(\left(\mathbb{I}_{\Lambda_{L}}^{(\omega, \mathcal{E})}\right)^{2} \mathrm{e}^{s\left|\Lambda_{L}\right| \mathbb{I}_{\Lambda_{L}}^{(\omega, \mathcal{E})}}\right) \varrho^{(\omega)}\left(\mathrm{e}^{s\left|\Lambda_{L}\right| \mathbb{I}_{\Lambda_{L}}^{(\omega, \mathcal{E})}}\right)-\left(\varrho^{(\omega)}\left(\mathbb{I}_{\Lambda_{L}}^{(\omega, \mathcal{E})} \mathrm{e}^{s\left|\Lambda_{L}\right| \mathbb{I}_{\Lambda_{L}}^{(\omega, \mathcal{E})}}\right)\right)^{2}}{\left(\varrho^{(\omega)}\left(\mathrm{e}^{s\left|\Lambda_{L}\right| \mathbb{I}_{\Lambda_{L}}^{(\omega, \mathcal{E})}}\right)\right)^{2}}\right) .
\end{aligned}
$$

See (32). Note that the above limits for the first- and second-order derivatives do not need to be taken only along subsequences, by the (Arzelà-) Ascoli theorem [19, Theorem A5] and (53). In particular, for $s=0$,

$$
\left.\partial_{s} J^{(s \mathcal{E})}\right|_{s=0}=\lim _{L \rightarrow \infty} \mathbb{E}\left[\varrho^{(\cdot)}\left(\mathbb{I}_{\Lambda_{L}}^{(\cdot, \mathcal{E})}\right)\right]=\lim _{L \rightarrow \infty} \varrho^{(\omega)}\left(\mathbb{I}_{\Lambda_{L}}^{(\omega, \mathcal{E})}\right)
$$

and

$$
\begin{aligned}
\left.\partial_{s}^{2} J^{(s \mathcal{E})}\right|_{s=0} & =\lim _{L \rightarrow \infty}\left|\Lambda_{L}\right| \mathbb{E}\left[\varrho^{(\cdot)}\left(\left(\mathbb{I}_{\Lambda_{L}^{(\cdot, \mathcal{E})}}\right)^{2}\right)-\left(\varrho^{(\cdot)}\left(\mathbb{I}_{\Lambda_{L}^{(\cdot, \mathcal{E})}}^{(j)}\right)^{2}\right]\right. \\
& =\lim _{L \rightarrow \infty}\left|\Lambda_{L}\right|\left(\varrho^{(\omega)}\left(\left(\mathbb{I}_{\Lambda_{L}^{(\omega, \mathcal{E})}}\right)^{2}\right)-\left(\varrho^{(\omega)}\left(\mathbb{I}_{\Lambda_{L}}^{(\omega, \mathcal{E})}\right)\right)^{2}\right) .
\end{aligned}
$$

By Equations (18)- 19 and $(56),\left.\partial_{s}^{2} \mathrm{~J}^{(s \mathcal{E})}\right|_{s=0}$ is the thermodynamic limit of the quantum fluctuations of linear response currents. 
From the proof of Proposition 4.1, it is apparent that the $n$-th derivative $\partial_{s}^{n} \mathrm{~J}_{\mathcal{Z}, \mathcal{Z}(\varrho), \mathcal{Z}(\tau)}^{(\omega,)}$, $n \in \mathbb{N}$, has the following structure:

$$
\begin{aligned}
\partial_{s}^{n} \mathrm{~J}_{\mathcal{Z}, \mathcal{Z}(\mathfrak{Q}), \mathcal{Z}(\tau)}^{(\omega, \mathcal{E})}= & \frac{1}{|\cup \mathcal{Z}|} \sum_{k=1}^{n} \sum_{x_{k}, y_{k} \in \mathbb{Z}^{d}}\left\langle\mathfrak{e}_{x_{1}}, K_{\mathcal{Z}, \mathcal{Z}(\tau)}^{(\omega, \mathcal{E})} \mathfrak{e}_{y_{1}}\right\rangle_{\mathfrak{h}} \cdots\left\langle\mathfrak{e}_{x_{k}}, K_{\mathcal{Z}, \mathcal{Z}(\tau)}^{(\omega, \mathcal{E})} \mathfrak{e}_{y_{k}}\right\rangle_{\mathfrak{h}} \\
& \times \varpi_{s}^{T}\left(a^{*}\left(\mathfrak{e}_{x_{1}}\right) a\left(\mathfrak{e}_{y_{1}}\right) ; \cdots ; a^{*}\left(\mathfrak{e}_{x_{n}}\right) a\left(\mathfrak{e}_{x_{n}}\right)\right) \\
= & \frac{1}{|\cup \mathcal{Z}|} \sum_{g \in \mathcal{G}_{n}^{c}} \sum_{k=1}^{n} \sum_{x_{k}, y_{k} \in \mathbb{Z}^{d}} \operatorname{sign}(g)\left\langle\mathfrak{e}_{x_{1}}, K_{\mathcal{Z}, \mathcal{Z}(\tau)}^{(\omega, \mathcal{E})} \mathfrak{e}_{y_{1}}\right\rangle_{\mathfrak{h}} \cdots\left\langle\mathfrak{e}_{x_{k}}, K_{\mathcal{Z}, \mathcal{Z}(\tau)}^{(\omega, \mathcal{E})} \mathfrak{e}_{y_{k}}\right\rangle_{\mathfrak{h}} \\
& \times \prod_{l \in g} k_{s}\left(l ; x_{1}, y_{1}, \ldots, x_{n}, y_{n}\right)
\end{aligned}
$$

where $\mathcal{G}_{n}^{c}$ is the set of all connected oriented graphs $g$ such that, for each vertex $v \in\{1, \ldots, n\}$ of $g \in \mathcal{G}_{n}^{c}$, there is exactly one line of the form $\left(v, \tilde{v}_{1}\right) \in g$ and exactly one line of the form $\left(\tilde{v}_{2}, v\right) \in g$, for some $\tilde{v}_{1}, \tilde{v}_{2} \in\{1, \ldots, n\}$. The constants $k_{s}\left(l ; x_{1}, y_{1}, \ldots, x_{n}, y_{n}\right), l \in\{1, \ldots, n\}^{2}$, $x_{1}, y_{1}, \ldots, x_{n}, y_{n} \in \mathbb{Z}^{d}$, are defined by

$$
k_{s}\left((i, j) ; x_{1}, y_{1}, \ldots, x_{n}, y_{n}\right) \doteq \begin{cases}\varpi_{s}\left(a^{*}\left(\mathfrak{e}_{x_{i}}\right) a\left(\mathfrak{e}_{y_{j}}\right)\right) & \text { if } i \leq j \\ \varpi_{s}\left(a\left(\mathfrak{e}_{y_{j}}\right) a^{*}\left(\mathfrak{e}_{x_{i}}\right)\right) & \text { if } i>j\end{cases}
$$

The quantity $\operatorname{sign}(g) \in\{-1,1\}$ is a sign only depending on the graph $g \in \mathcal{G}_{n}^{c}$. By using this expression, exactly as in the special case $n=3$, for any fixed $n \in \mathbb{N}$ and electric field $\mathcal{E}$ one can bound the $n$-th derivative $\partial_{s}^{n} \mathrm{~J}_{\mathcal{Z}, \mathcal{Z}^{(e)}, \mathcal{Z}^{(\tau)}}^{(\omega \mathcal{B})}$ uniformly. This implies that the generating function $s \mapsto \mathrm{J}^{(s \mathcal{E})}$ defined by 16 is a smooth function of $s \in \mathbb{R}$, by the (Arzelà-) Ascoli theorem [19, Theorem A5] used as in the proof of Corollary 4.2. We refrain from working out the full arguments to prove this claim since absolutely no new conceptual ingredient would appear in this generalization.

\subsection{Non-Vanishing Second Derivative of Generating Functions at the Origin}

We discuss necessary conditions for

$$
\left.\partial_{s}^{2} \mathrm{~J}^{(s \mathcal{E})}\right|_{s=0} \neq 0
$$

which is a condition appearing in Theorem 3.1 (ii). In other words, the aim of this section is to prove Theorem 3.3 . To this end, it is convenient to write this quantity by means of the one-particle Hilbert space $\mathfrak{h}$.

\section{Lemma 4.3 (Quantum fluctuations on the one-particle Hilbert space)}

For all $\beta \in \mathbb{R}^{+}, \vartheta, \lambda \in \mathbb{R}_{0}^{+}, \mathcal{E} \in C_{0}^{0}\left(\mathbb{R} ; \mathbb{R}^{d}\right)$ and $\vec{w} \in \mathbb{R}^{d}$ with $\|\vec{w}\|_{\mathbb{R}^{d}}=1$,

$$
\left.\partial_{s}^{2} \mathrm{~J}^{(s \mathcal{E})}\right|_{s=0}=\lim _{L \rightarrow \infty} \frac{1}{\left|\Lambda_{L}\right|} \mathbb{E}\left[\operatorname{Tr}_{\mathfrak{h}}\left(K_{\left\{\Lambda_{L}\right\},\left\{\mathbb{Z}^{d}\right\}}^{(\cdot \mathcal{E})} \frac{1}{1+\mathrm{e}^{-\beta h^{(\cdot)}}} K_{\left\{\Lambda_{L}\right\},\left\{\mathbb{Z}^{d}\right\}}^{(\cdot, \mathcal{E})} \frac{1}{1+\mathrm{e}^{\beta h^{(\cdot)}}}\right)\right]
$$

with $\operatorname{Tr}_{\mathfrak{h}}$ being the trace on $\mathfrak{h} \doteq \ell^{2}\left(\mathbb{Z}^{d} ; \mathbb{C}\right)$.

Proof. Fix all parameters of the lemma. Using Equations (32) and (35) together with the quasi-free property of $\varrho^{(\omega)}$, one obtains from $(56)$ that

$$
\begin{aligned}
\left.\partial_{s}^{2} \mathrm{~J}^{(s \mathcal{E})}\right|_{s=0}=\lim _{L \rightarrow \infty} \frac{1}{\left|\Lambda_{L}\right|} \sum_{x, y, u, v \in \mathbb{Z}^{d}}\left\langle\mathfrak{e}_{x}, K_{\left\{\Lambda_{L}\right\},\left\{\mathbb{Z}^{d}\right\}}^{(\omega, \mathcal{E})} \mathfrak{e}_{y}\right\rangle_{\mathfrak{h}}\left\langle\mathfrak{e}_{u}, K_{\left\{\Lambda_{L}\right\},\left\{\mathbb{Z}^{d}\right\}}^{(\omega, \mathcal{E})} \boldsymbol{e}_{v}\right\rangle_{\mathfrak{h}} \\
\\
\times \varrho^{(\omega)}\left(a\left(\mathfrak{e}_{y}\right) a\left(\mathfrak{e}_{u}\right)^{*}\right) \varrho^{(\omega)}\left(a\left(\mathfrak{e}_{x}\right)^{*} a\left(\mathfrak{e}_{v}\right)\right),
\end{aligned}
$$


because of the identity

$$
\rho\left(a\left(\mathfrak{e}_{x}\right)^{*} a\left(\mathfrak{e}_{y}\right) a\left(\mathfrak{e}_{u}\right)^{*} a\left(\mathfrak{e}_{v}\right)\right)=\rho\left(a\left(\mathfrak{e}_{x}\right)^{*} a\left(\mathfrak{e}_{y}\right)\right) \rho\left(a\left(\mathfrak{e}_{u}\right)^{*} a\left(\mathfrak{e}_{v}\right)\right)+\rho\left(a\left(\mathfrak{e}_{y}\right) a\left(\mathfrak{e}_{u}\right)^{*}\right) \rho\left(a\left(\mathfrak{e}_{x}\right)^{*} a\left(\mathfrak{e}_{v}\right)\right)
$$

for any $x, y, u, v \in \mathbb{Z}^{d}$ and quasi-free state $\rho$ on $\mathcal{U}$, see (4) and (8). By Equation (6) and straightforward computations, the assertion follows.

Therefore, (57) holds true if

$$
\lim _{L \rightarrow \infty}\left\{\frac{1}{\left|\Lambda_{L}\right|}\left|\operatorname{Tr}_{\mathfrak{h}}\left(K_{\left\{\Lambda_{L}\right\},\left\{\mathbb{Z}^{d}\right\}}^{(\omega, \mathcal{E})} \frac{1}{1+\mathrm{e}^{-\beta h^{(\omega)}}} K_{\left\{\Lambda_{L}\right\},\left\{\mathbb{Z}^{d}\right\}}^{(\omega, \mathcal{E})} \frac{1}{1+\mathrm{e}^{\beta h^{(\omega)}}}\right)\right|\right\} \geq \varepsilon>0
$$

for some strictly positive constant $\varepsilon \in \mathbb{R}^{+}$. In order to verify this bound, we start with an elementary observation:

Lemma 4.4 (Quantum fluctuations and the Hilbert-Schmidt norm of $K_{\left\{\Lambda_{L}\right\},\left\{\mathbb{Z}^{d}\right\}}^{(\omega, \mathcal{E})}$ ) For all $\beta \in \mathbb{R}^{+}, \vartheta, \lambda \in \mathbb{R}_{0}^{+}, \omega \in \Omega, \mathcal{E} \in C_{0}^{0}\left(\mathbb{R} ; \mathbb{R}^{d}\right)$ and $\vec{w} \in \mathbb{R}^{d}$ with $\|\vec{w}\|_{\mathbb{R}^{d}}=1$,

$$
\begin{aligned}
\operatorname{Tr}_{\mathfrak{h}}\left(K_{\left\{\Lambda_{L}\right\},\left\{\mathbb{Z}^{d}\right\}}^{(\omega, \mathcal{E})} \frac{1}{1+\mathrm{e}^{-\beta h^{(\omega)}}} K_{\left\{\Lambda_{L}\right\},\left\{\mathbb{Z}^{d}\right\}}^{(\omega, \mathcal{E})} \frac{1}{1+\mathrm{e}^{\beta h^{(\omega)}}}\right) \\
\geq \frac{1}{\left(1+\mathrm{e}^{\beta(2 d(2+\vartheta)+\lambda)}\right)^{2}} \operatorname{Tr}_{\mathfrak{h}}\left(\left(K_{\left\{\Lambda_{L}\right\},\left\{\mathbb{Z}^{d}\right\}}^{(\omega, \mathcal{E})}\right)^{*} K_{\left\{\Lambda_{L}\right\},\left\{\mathbb{Z}^{d}\right\}}^{(\omega, \mathcal{E})}\right) .
\end{aligned}
$$

Proof. Fix all parameters of the lemma. By the functional calculus, $\left(1+\mathrm{e}^{ \pm \beta h^{(\omega)}}\right)^{-1}$ are positive operators satisfying

$$
\frac{1}{1+\mathrm{e}^{ \pm \beta h^{(\omega)}}} \geq \frac{1}{1+\mathrm{e}^{\beta \sup _{\omega \in \Omega}\left\|h^{(\omega)}\right\|_{\mathcal{B}(\mathfrak{h})}}} \mathbf{1}_{\mathfrak{h}},
$$

while, for any $\omega=\left(\omega_{1}, \omega_{2}\right) \in \Omega$ and $\lambda, \vartheta \in \mathbb{R}_{0}^{+}$,

$$
\left\|h^{(\omega)}\right\|_{\mathcal{B}(\mathfrak{h})} \leq\left\|\Delta_{\omega, \vartheta}\right\|_{\mathcal{B}(\mathfrak{h})}+\lambda\left\|\omega_{1}\right\|_{\mathcal{B}(\mathfrak{h})} \leq 2 d(2+\vartheta)+\lambda
$$

see 22- -3 . Since $K_{\left\{\Lambda_{L}\right\},\left\{\mathbb{Z}^{d}\right\}}^{(\omega, \mathcal{E})}$ is a self-adjoint operator (see 36 or 59 below), it thus suffices to use the cyclicity of the trace to prove the lemma.

Recall that $K_{\left\{\Lambda_{L}\right\},\left\{\mathbb{Z}^{d}\right\}}^{(\omega, \mathcal{E})}$ is defined by 36$)$, that is in this case,

$$
K_{\left\{\Lambda_{L}\right\},\left\{\mathbb{Z}^{d}\right\}}^{(\omega, \mathcal{E})} \doteq \sum_{k, q=1}^{d} w_{k} \int_{-\infty}^{0}\{\mathcal{E}(\alpha)\}_{q}\left(\delta_{k, q} \mathbf{M}_{k}^{(L, \omega)}+\int_{0}^{-\alpha} \mathbf{N}_{\gamma, q, k}^{(L, \omega)} \mathrm{d} \gamma\right) \mathrm{d} \alpha
$$

where, for any $k, q \in\{1, \ldots, d\}, \gamma \in \mathbb{R}, \vartheta, \lambda \in \mathbb{R}_{0}^{+}, \omega \in \Omega$ and $L \in \mathbb{R}^{+}$,

$$
\begin{aligned}
\mathbf{M}_{k}^{(L, \omega)} & \doteq \sum_{x, x+e_{k} \in \Lambda_{L}} 2 \Re \mathrm{e}\left\{S_{x+e_{k}, x}^{(\omega)}\right\} \\
\mathbf{N}_{\gamma, q, k}^{(L, \omega)} & \doteq \sum_{x, y, x+e_{k}, y+e_{q} \in \Lambda_{L}} 4 i\left[\mathrm{e}^{-i \gamma h^{(\omega)}} \Im \mathrm{m}\left\{S_{y+e_{q}, y}^{(\omega)}\right\} \mathrm{e}^{i \gamma h^{(\omega)}}, \Im \mathrm{m}\left\{S_{x+e_{k}, x}^{(\omega)}\right\}\right]
\end{aligned}
$$

with $S_{x, y}^{(\omega)}$ being the single-hopping operators defined by 33 - 34 for any $x, y \in \mathbb{Z}^{d}$.

The square of the Hilbert-Schmidt norm of $K_{\left\{\Lambda_{L}\right\},\left\{\mathbb{Z}^{d}\right\}}^{(\omega, \mathcal{E}}$ is obviously equal to

$$
\operatorname{Tr}_{\mathfrak{h}}\left(\left(K_{\left\{\Lambda_{L}\right\},\left\{\mathbb{Z}^{d}\right\}}^{(\omega, \mathcal{E})}\right)^{*} K_{\left\{\Lambda_{L}\right\},\left\{\mathbb{Z}^{d}\right\}}^{(\omega, \mathcal{E})}\right)=\sum_{z \in \mathbb{Z}^{d}}\left\|K_{\left\{\Lambda_{L}\right\},\left\{\mathbb{Z}^{d}\right\}}^{(\omega, \mathcal{E})} \mathfrak{e}_{z}\right\|_{\mathfrak{h}}^{2}
$$

and, consequently, we derive an explicit expression for the vectors

$$
K_{\left\{\Lambda_{L}\right\},\left\{\mathbb{Z}^{d}\right\}}^{(\omega, \mathcal{E})} \mathfrak{e}_{z} \in \mathfrak{h}, \quad z \in \mathbb{Z}^{d} .
$$

This can be directly obtained from Equation 59 together with the following assertion: 
Lemma 4.5 (Explicit computations of $\mathbf{M}_{k}^{(L, \omega)}$ and $\mathbf{N}_{\gamma, q, k}^{(L, \omega)}$ in the canonical basis) For all $k, q \in\{1, \ldots, d\}, \gamma \in \mathbb{R}, \vartheta, \lambda \in \mathbb{R}_{0}^{+}, \omega \in \Omega, \gamma \in \mathbb{R}, L \geq 2$ and $z \in \Lambda_{L / 2}$,

$$
\mathbf{M}_{k}^{(L, \omega)} \mathfrak{e}_{z}=\left\langle\mathfrak{e}_{z-e_{k}}, \Delta_{\omega, \vartheta} \mathfrak{e}_{z}\right\rangle_{\mathfrak{h}} \mathfrak{e}_{z-e_{k}}+\left\langle\mathfrak{e}_{z+e_{k}}, \Delta_{\omega, \vartheta} \mathfrak{e}_{z}\right\rangle_{\mathfrak{h}} \mathfrak{e}_{z+e_{k}}
$$

and, in the limit $L \rightarrow \infty$,

$$
\mathbf{N}_{\gamma, q, k}^{(L, \omega)} \mathfrak{e}_{z}=\sum_{x, y \in \mathbb{Z}^{d}} \zeta_{x, y, z} \mathfrak{e}_{x}+\mathbf{R}_{\gamma, q, k}^{(L, \omega)} \mathfrak{e}_{z}, \quad \sum_{x, y \in \mathbb{Z}^{d}}\left|\zeta_{x, y, z}\right|^{2}<\infty,
$$

with $\mathbf{R}_{\gamma, q, k}^{(L, \omega)} \in \mathcal{B}(\mathfrak{h})$ satisfying

$$
\lim _{L \rightarrow \infty}\left\|\mathbf{R}_{\gamma, q, k}^{(L, \omega)}\right\|_{\mathcal{B}(\mathfrak{h})}=0
$$

uniformly with respect to $\omega \in \Omega, \lambda \in \mathbb{R}_{0}^{+}$and $\vartheta, \gamma$ in compact subsets of $\mathbb{R}_{0}^{+}$and $\mathbb{R}$, respectively, and where, for any $x, y, z \in \mathbb{Z}^{d}$,

$$
\begin{aligned}
\zeta_{x, y, z} \doteq & i\left(1+\vartheta \omega_{2}\left(\left\{x-e_{k}, x\right\}\right)\right)\left(1+\vartheta \omega_{2}\left(\left\{y, y+e_{q}\right\}\right)\right)\left\langle\mathfrak{e}_{x-e_{k}}, \mathrm{e}^{-i \gamma h^{(\omega)}} \mathfrak{e}_{y+e_{q}}\right\rangle_{\mathfrak{h}}\left\langle\mathfrak{e}_{y}, \mathrm{e}^{i \gamma h^{(\omega)}} \mathfrak{e}_{z}\right\rangle_{\mathfrak{h}} \\
& -i\left(1+\vartheta \omega_{2}\left(\left\{x-e_{k}, x\right\}\right)\right)\left(1+\vartheta \overline{\omega_{2}\left(\left\{y+e_{q}, y\right\}\right)}\right)\left\langle\mathfrak{e}_{x-e_{k}}, \mathrm{e}^{-i \gamma h^{(\omega)}} \mathfrak{e}_{y}\right\rangle_{\mathfrak{h}}\left\langle\mathfrak{e}_{y+e_{q}}, \mathrm{e}^{i \gamma h^{(\omega)}} \mathfrak{e}_{z}\right\rangle_{\mathfrak{h}} \\
& -i\left(1+\vartheta \overline{\omega_{2}\left(\left\{x+e_{k}, x\right\}\right)}\right)\left(1+\vartheta \omega_{2}\left(\left\{y, y+e_{q}\right\}\right)\right)\left\langle\mathfrak{e}_{x+e_{k}}, \mathrm{e}^{-i \gamma h^{(\omega)}} \mathfrak{e}_{y+e_{q}}\right\rangle_{\mathfrak{h}}\left\langle\mathfrak{e}_{y}, \mathrm{e}^{i \gamma h^{(\omega)}} \mathfrak{e}_{z}\right\rangle_{\mathfrak{h}} \\
& +i\left(1+\vartheta \overline{\omega_{2}\left(\left\{x+e_{k}, x\right\}\right)}\right)\left(1+\vartheta \overline{\omega_{2}\left(\left\{y+e_{q}, y\right\}\right)}\left\langle\mathfrak{e}_{x+e_{k}}, \mathrm{e}^{-i \gamma h^{(\omega)}} \mathfrak{e}_{y}\right\rangle_{\mathfrak{h}}\left\langle\mathfrak{e}_{y+e_{q}}, \mathrm{e}^{i \gamma h^{(\omega)}} \mathfrak{e}_{z}\right\rangle_{\mathfrak{h}}\right. \\
& -i\left(1+\vartheta \omega_{2}\left(\left\{y, y+e_{q}\right\}\right)\right)\left(1+\vartheta \omega_{2}\left(\left\{z, z+e_{k}\right\}\right)\right)\left\langle\mathfrak{e}_{y}, \mathrm{e}^{i \gamma h^{(\omega)}} \mathfrak{e}_{z+e_{k}}\right\rangle_{\mathfrak{h}}\left\langle\mathfrak{e}_{x}, \mathrm{e}^{-i \gamma h^{(\omega)}} \mathfrak{e}_{y+e_{q}}\right\rangle_{\mathfrak{h}} \\
& +i\left(1+\vartheta \omega_{2}\left(\left\{y, y+e_{q}\right\}\right)\right)\left(1+\vartheta \omega_{2}\left(\left\{z, z-e_{k}\right\}\right)\right)\left\langle\mathfrak{e}_{y}, \mathrm{e}^{i \gamma h^{(\omega)}} \mathfrak{e}_{z-e_{k}}\right\rangle_{\mathfrak{h}}\left\langle\mathfrak{e}_{x}, \mathrm{e}^{-i \gamma h^{(\omega)}} \mathfrak{e}_{y+e_{q}}\right\rangle_{\mathfrak{h}} \\
& +i\left(1+\vartheta \overline{\omega_{2}\left(\left\{y+e_{q}, y\right\}\right)}\right)\left(1+\vartheta \omega_{2}\left(\left\{z, z+e_{k}\right\}\right)\right)\left\langle\mathfrak{e}_{y+e_{q}}, \mathrm{e}^{i \gamma h^{(\omega)}} \mathfrak{e}_{z+e_{k}}\right\rangle_{\mathfrak{h}}\left\langle\mathfrak{e}_{x}, \mathrm{e}^{-i \gamma h^{(\omega)}} \mathfrak{e}_{y}\right\rangle_{\mathfrak{h}} \\
& -i\left(1+\vartheta \overline{\omega_{2}\left(\left\{y+e_{q}, y\right\}\right)}\right)\left(1+\vartheta \overline{\omega_{2}\left(\left\{z, z-e_{k}\right\}\right)}\right)\left\langle\mathfrak{e}_{y+e_{q}}, \mathrm{e}^{i \gamma h^{(\omega)}} \mathfrak{e}_{z-e_{k}}\right\rangle_{\mathfrak{h}}\left\langle\mathfrak{e}_{x}, \mathrm{e}^{-i \gamma h^{(\omega)}} \mathfrak{e}_{y}\right\rangle_{\mathfrak{h}} .
\end{aligned}
$$

Proof. Fix in all the proof $k, q \in\{1, \ldots, d\}, \vartheta, \lambda \in \mathbb{R}_{0}^{+}, \omega \in \Omega, \gamma \in \mathbb{R}, L \geq 2$ and $z \in \Lambda_{L / 2}$. Since, by (33)-34), for any $x, y \in \mathbb{Z}^{d}$,

$$
2 \Re \mathrm{e}\left\{S_{x, y}^{(\omega)}\right\}=\left\langle\mathfrak{e}_{y}, \Delta_{\omega, \vartheta} \mathfrak{e}_{x}\right\rangle_{\mathfrak{h}} P_{\{y\}} s_{y-x} P_{\{x\}}+\left\langle\mathfrak{e}_{x}, \Delta_{\omega, \vartheta} \mathfrak{e}_{y}\right\rangle_{\mathfrak{h}} P_{\{x\}} s_{x-y} P_{\{y\}},
$$

we deduce from $(60)$ together with $(28)$ and $(33)$ that

$$
\begin{aligned}
\mathbf{M}_{k}^{(L, \omega)} \mathfrak{e}_{z}= & \sum_{x, x+e_{k} \in \Lambda_{L}}\left(\delta_{z, x+e_{k}}\left\langle\mathfrak{e}_{x}, \Delta_{\omega, \vartheta} \mathfrak{e}_{x+e_{k}}\right\rangle_{\mathfrak{h}} \mathfrak{e}_{x}+\delta_{z, x}\left\langle\mathfrak{e}_{x+e_{k}}, \Delta_{\omega, \vartheta} \mathfrak{e}_{x}\right\rangle_{\mathfrak{h}} \mathfrak{e}_{x+e_{k}}\right) \\
= & \mathbf{1}\left[z \in \Lambda_{L}\right] \mathbf{1}\left[\left(z-e_{k}\right) \in \Lambda_{L}\right]\left\langle\mathfrak{e}_{z-e_{k}}, \Delta_{\omega, \vartheta} \mathfrak{e}_{z}\right\rangle_{\mathfrak{h}} \mathfrak{e}_{z-e_{k}} \\
& +\mathbf{1}\left[z \in \Lambda_{L}\right] \mathbf{1}\left[\left(z+e_{k}\right) \in \Lambda_{L}\right]\left\langle\mathfrak{e}_{z+e_{k}}, \Delta_{\omega, \vartheta} \mathfrak{e}_{z}\right\rangle_{\mathfrak{h}} \mathfrak{e}_{z+e_{k}} .
\end{aligned}
$$

If $z \in \Lambda_{L / 2} \subseteq \Lambda_{L}$ and $L \geq 2$ then, obviously, $z,\left(z-e_{k}\right),\left(z+e_{k}\right) \in \Lambda_{L}$ and the last equality yields the first assertion.

Since, again by (33)-34, for any $x, y \in \mathbb{Z}^{d}$,

$$
2 \Im \mathrm{mm}\left\{S_{x, y}^{(\omega)}\right\}=i\left(\left\langle\mathfrak{e}_{y}, \Delta_{\omega, \vartheta} \mathfrak{e}_{x}\right\rangle_{\mathfrak{h}} P_{\{y\}} s_{y-x} P_{\{x\}}-\left\langle\mathfrak{e}_{x}, \Delta_{\omega, \vartheta} \mathfrak{e}_{y}\right\rangle_{\mathfrak{h}} P_{\{x\}} s_{x-y} P_{\{y\}}\right),
$$


we compute that, for any $x, y \in \mathbb{Z}^{d}$,

$$
\begin{aligned}
& 4 i\left[\mathrm{e}^{-i \gamma h^{(\omega)}} \Im \mathrm{m}\left\{S_{y+e_{q}, y}^{(\omega)}\right\} \mathrm{e}^{i \gamma h^{(\omega)}}, \Im \mathrm{m}\left\{S_{x+e_{k}, x}^{(\omega)}\right\}\right] \\
= & i\left\langle\mathfrak{e}_{x+e_{k}}, \Delta_{\omega, \vartheta} \mathfrak{e}_{x}\right\rangle_{\mathfrak{h}}\left\langle\mathfrak{e}_{y+e_{q}}, \Delta_{\omega, \vartheta} \mathfrak{e}_{y}\right\rangle_{\mathfrak{h}} s_{e_{k}} P_{\{x\}} \mathrm{e}^{-i \gamma h^{(\omega)}} s_{e_{q}} P_{\{y\}} \mathrm{e}^{i \gamma h^{(\omega)}} \\
& -i\left\langle\mathfrak{e}_{x+e_{k}}, \Delta_{\omega, \vartheta} \mathfrak{e}_{x}\right\rangle_{\mathfrak{h}}\left\langle\mathfrak{e}_{y}, \Delta_{\omega, \vartheta} \mathfrak{e}_{y+e_{q}}\right\rangle_{\mathfrak{h}} s_{e_{k}} P_{\{x\}} \mathrm{e}^{-i \gamma h^{(\omega)}} s_{-e_{q}} P_{\left\{y+e_{q}\right\}} \mathrm{e}^{i \gamma h^{(\omega)}} \\
& -i\left\langle\mathfrak{e}_{x}, \Delta_{\omega, \vartheta} \mathfrak{e}_{x+e_{k}}\right\rangle_{\mathfrak{h}}\left\langle\mathfrak{e}_{y+e_{q}}, \Delta_{\omega, \vartheta} \mathfrak{e}_{y}\right\rangle_{\mathfrak{h}} s_{-e_{k}} P_{\left\{x+e_{k}\right\}} \mathrm{e}^{-i \gamma h^{(\omega)}} s_{e_{q}} P_{\{y\}} \mathrm{e}^{i \gamma h^{(\omega)}} \\
& +i\left\langle\mathfrak{e}_{x}, \Delta_{\omega, \vartheta} \mathfrak{e}_{x+e_{k}}\right\rangle_{\mathfrak{h}}\left\langle\mathfrak{e}_{y}, \Delta_{\omega, \vartheta} \mathfrak{e}_{y+e_{q}}\right\rangle_{\mathfrak{h}} s_{-e_{k}} P_{\left\{x+e_{k}\right\}} \mathrm{e}^{-i \gamma h^{(\omega)}} s_{-e_{q}} P_{\left\{y+e_{q}\right\}} \mathrm{e}^{i \gamma h^{(\omega)}} \\
& -i\left\langle\mathfrak{e}_{y+e_{q}}, \Delta_{\omega, \vartheta} \mathfrak{e}_{y}\right\rangle_{\mathfrak{h}}\left\langle\mathfrak{e}_{x+e_{k}}, \Delta_{\omega, \vartheta} \mathfrak{e}_{x}\right\rangle_{\mathfrak{h}} \mathrm{e}^{-i \gamma h^{(\omega)}} s_{e_{q}} P_{\{y\}} \mathrm{e}^{i \gamma h^{(\omega)}} s_{e_{k}} P_{\{x\}} \\
& +i\left\langle\mathfrak{e}_{y+e_{q}}, \Delta_{\omega, \vartheta} \mathfrak{e}_{y}\right\rangle_{\mathfrak{h}}\left\langle\mathfrak{e}_{x}, \Delta_{\omega, \vartheta} \mathfrak{e}_{x+e_{k}}\right\rangle_{\mathfrak{h}} \mathrm{e}^{-i \gamma h^{(\omega)}} s_{e_{q}} P_{\{y\}} \mathrm{e}^{i \gamma h(\omega)} s_{-e_{k}} P_{\left\{x+e_{k}\right\}} \\
& +i\left\langle\mathfrak{e}_{y}, \Delta_{\omega, \vartheta} \mathfrak{e}_{y+e_{q}}\right\rangle_{\mathfrak{h}}\left\langle\mathfrak{e}_{x+e_{k}}, \Delta_{\omega, \vartheta} \mathfrak{e}_{x}\right\rangle_{\mathfrak{h}} \mathrm{e}^{-i \gamma h^{(\omega)}} s_{-e_{q}} P_{\left\{y+e_{q}\right\}} \mathrm{e}^{i \gamma h^{(\omega)}} s_{e_{k}} P_{\{x\}} \\
& -i\left\langle\mathfrak{e}_{y}, \Delta_{\omega, \vartheta} \mathfrak{e}_{y+e_{q}}\right\rangle_{\mathfrak{h}}\left\langle\mathfrak{e}_{x}, \Delta_{\omega, \vartheta} \mathfrak{e}_{x+e_{k}}\right\rangle_{\mathfrak{h}} \mathrm{e}^{-i \gamma h^{(\omega)}} s_{-e_{q}} P_{\left\{y+e_{q}\right\}} \mathrm{e}^{i \gamma h^{(\omega)}} s_{-e_{k}} P_{\left\{x+e_{k}\right\}} .
\end{aligned}
$$

Using this last equality together with (33)- (34) and (61), we thus get that

$$
\begin{aligned}
\mathbf{N}_{\gamma, q, k}^{(L, \omega)} \mathfrak{e}_{z}= & \sum_{x, y, x+e_{k}, y+e_{q} \in \Lambda_{L}} \\
& \left\{i\left\langle\mathfrak{e}_{x+e_{k}}, \Delta_{\omega, \vartheta} \mathfrak{e}_{x}\right\rangle_{\mathfrak{h}}\left\langle\mathfrak{e}_{y+e_{q}}, \Delta_{\omega, \vartheta} \mathfrak{e}_{y}\right\rangle_{\mathfrak{h}}\left\langle\mathfrak{e}_{x}, \mathrm{e}^{-i \gamma h^{(\omega)}} \mathfrak{e}_{y+e_{q}}\right\rangle_{\mathfrak{h}}\left\langle\mathfrak{e}_{y}, \mathrm{e}^{i \gamma h^{(\omega)}} \mathfrak{e}_{z}\right\rangle_{\mathfrak{h}} \mathfrak{e}_{x+e_{k}}\right. \\
& -i\left\langle\mathfrak{e}_{x+e_{k}}, \Delta_{\omega, \vartheta} \mathfrak{e}_{x}\right\rangle_{\mathfrak{h}}\left\langle\mathfrak{e}_{y}, \Delta_{\omega, \vartheta} \mathfrak{e}_{y+e_{q}}\right\rangle_{\mathfrak{h}}\left\langle\mathfrak{e}_{x}, \mathrm{e}^{-i \gamma h^{(\omega)}} \mathfrak{e}_{y}\right\rangle_{\mathfrak{h}}\left\langle\mathfrak{e}_{y+e_{q}}, \mathrm{e}^{i \gamma h^{(\omega)}} \mathfrak{e}_{z}\right\rangle_{\mathfrak{h}} \mathfrak{e}_{x+e_{k}} \\
& -i\left\langle\mathfrak{e}_{x}, \Delta_{\omega, \vartheta} \mathfrak{e}_{x+e_{k}}\right\rangle_{\mathfrak{h}}\left\langle\mathfrak{e}_{y+e_{q}}, \Delta_{\omega, \vartheta} \mathfrak{e}_{y}\right\rangle_{\mathfrak{h}}\left\langle\mathfrak{e}_{x+e_{k}}, \mathrm{e}^{-i \gamma h^{(\omega)}} \mathfrak{e}_{y+e_{q}}\right\rangle_{\mathfrak{h}}\left\langle\mathfrak{e}_{y}, \mathrm{e}^{i \gamma h^{(\omega)}} \mathfrak{e}_{z}\right\rangle_{\mathfrak{h}} \mathfrak{e}_{x} \\
& +i\left\langle\mathfrak{e}_{x}, \Delta_{\omega, \vartheta} \mathfrak{e}_{x+e_{k}}\right\rangle_{\mathfrak{h}}\left\langle\mathfrak{e}_{y}, \Delta_{\omega, \vartheta} \mathfrak{e}_{y+e_{q}}\right\rangle_{\mathfrak{h}}\left\langle\mathfrak{e}_{x+e_{k}}, \mathrm{e}^{-i \gamma h^{(\omega)}} \mathfrak{e}_{y}\right\rangle_{\mathfrak{h}}\left\langle\mathfrak{e}_{y+e_{q}}, \mathrm{e}^{i \gamma h^{(\omega)}} \mathfrak{e}_{z}\right\rangle_{\mathfrak{h}} \mathfrak{e}_{x} \\
& -i \delta_{x, z}\left\langle\mathfrak{e}_{y+e_{q}}, \Delta_{\omega, \vartheta} \mathfrak{e}_{y}\right\rangle_{\mathfrak{h}}\left\langle\mathfrak{e}_{x+e_{k}}, \Delta_{\omega, \vartheta} \mathfrak{e}_{x}\right\rangle_{\mathfrak{h}}\left\langle\mathfrak{e}_{y}, \mathrm{e}^{i \gamma h^{(\omega)}} \mathfrak{e}_{x+e_{k}}\right\rangle_{\mathfrak{h}} \mathrm{e}^{-i \gamma h^{(\omega)}} \mathfrak{e}_{y+e_{q}} \\
& +i \delta_{x+e_{k}, z}\left\langle\mathfrak{e}_{y+e_{q}}, \Delta_{\omega, \vartheta} \mathfrak{e}_{y}\right\rangle_{\mathfrak{h}}\left\langle\mathfrak{e}_{x}, \Delta_{\omega, \vartheta} \mathfrak{e}_{x+e_{k}}\right\rangle_{\mathfrak{h}}\left\langle\mathfrak{e}_{y}, \mathrm{e}^{i \gamma h^{(\omega)}} \mathfrak{e}_{x}\right\rangle_{\mathfrak{h}} \mathrm{e}^{-i \gamma h^{(\omega)}} \mathfrak{e}_{y+e_{q}} \\
& +i \delta_{x, z}\left\langle\mathfrak{e}_{y}, \Delta_{\omega, \vartheta} \mathfrak{e}_{y+e_{q}}\right\rangle_{\mathfrak{h}}\left\langle\mathfrak{e}_{x+e_{k}}, \Delta_{\omega, \vartheta} \mathfrak{e}_{x}\right\rangle_{\mathfrak{h}}\left\langle\mathfrak{e}_{y+e_{q}}, \mathrm{e}^{i \gamma h^{(\omega)}} \mathfrak{e}_{x+e_{k}}\right\rangle_{\mathfrak{h}} \mathrm{e}^{-i \gamma h(\omega)} \mathfrak{e}_{y} \\
& \left.-i \delta_{x+e_{k}, z}\left\langle\mathfrak{e}_{y}, \Delta_{\omega, \vartheta} \mathfrak{e}_{y+e_{q}}\right\rangle_{\mathfrak{h}}\left\langle\mathfrak{e}_{x}, \Delta_{\omega, \vartheta} \mathfrak{e}_{x+e_{k}}\right\rangle_{\mathfrak{h}}\left\langle\mathfrak{e}_{y+e_{q}}, \mathrm{e}^{i \gamma h^{(\omega)}} \mathfrak{e}_{x}\right\rangle_{\mathfrak{h}} \mathrm{e}^{-i \gamma h^{(\omega)}} \mathfrak{e}_{y}\right\}
\end{aligned}
$$

By using (2) and (42)-(43) together with

$$
\sum_{z \in \mathbb{Z}^{d}} \mathrm{e}^{-2 \mu_{\eta}(|x-z|+|y-z|)} \leq \mathrm{e}^{-\mu_{\eta}|x-y|} \sum_{z \in \mathbb{Z}^{d}} \mathrm{e}^{-\mu_{\eta}(|x-z|+|y-z|)} \leq \mathrm{e}^{-\mu_{\eta}|x-y|} \sum_{z \in \mathbb{Z}^{d}} \mathrm{e}^{-2 \mu_{\eta}|z|} .
$$

(which are simple consequences of Cauchy-Schwarz and triangle inequalities), all the above summands are absolutely summable, uniformly with respect to $L \in \mathbb{R}^{+}, \omega \in \Omega, \lambda \in \mathbb{R}_{0}^{+}$ and $\vartheta, \gamma$ in compact subsets of $\mathbb{R}_{0}^{+}$and $\mathbb{R}$, respectively. For instance, for any (characteristic) functions $f, g: \mathbb{Z}^{d} \rightarrow\{0,1\}$, one estimates that

$$
\begin{gathered}
\sum_{x, y \in \mathbb{Z}^{d}} f(x)^{2} g(y)^{2}\left|\left\langle\mathfrak{e}_{x+e_{k}}, \Delta_{\omega, \vartheta} \mathfrak{e}_{x}\right\rangle_{\mathfrak{h}}\left\langle\mathfrak{e}_{y+e_{q}}, \Delta_{\omega, \vartheta} \mathfrak{e}_{y}\right\rangle_{\mathfrak{h}}\left\langle\mathfrak{e}_{x}, \mathrm{e}^{-i \gamma h^{(\omega)}} \mathfrak{e}_{y+e_{q}}\right\rangle_{\mathfrak{h}}\left\langle\mathfrak{e}_{y}, \mathrm{e}^{i \gamma h^{(\omega)}} \mathfrak{e}_{z}\right\rangle_{\mathfrak{h}}\right|\left\|\mathfrak{e}_{x+e_{k}}\right\|_{\mathfrak{h}} \\
\leq 36^{2}(1+\vartheta)^{2} \mathrm{e}^{2|\gamma \eta|} \sum_{x, y \in \mathbb{Z}^{d}} f(x)^{2} g(y)^{2} \mathrm{e}^{-2 \mu_{\eta}\left(\left|x-e_{q}-y\right|+|z-y|\right)} \\
\leq 36^{2}(1+\vartheta)^{2} \mathrm{e}^{2|\gamma \eta|}\left(\sum_{u \in \mathbb{Z}^{d}} g(u+z)^{2} \mathrm{e}^{-2 \mu_{\eta}|u|}\right)^{1 / 2} \\
\times \sum_{x \in \mathbb{Z}^{d}} f(x)^{2} \mathrm{e}^{-\mu_{\eta}\left|x-e_{q}-z\right|}\left(\sum_{y \in \mathbb{Z}^{d}} g\left(y+x-e_{q}\right)^{2} \mathrm{e}^{-2 \mu_{\eta}|y|}\right)^{1 / 2}<\infty .
\end{gathered}
$$


(Recall that $\mu_{\eta}>0$, by (43).) In fact, by the same arguments combined with

$$
\|C\|_{\mathcal{B}(\mathfrak{h})} \leq \sup _{x \in \mathbb{Z}^{d}} \sum_{z \in \mathbb{Z}^{d}}\left|\left\langle\mathfrak{e}_{x}, C \mathfrak{e}_{z}\right\rangle_{\mathfrak{h}}\right|, \quad C \in \mathcal{B}(\mathfrak{h}),
$$

(see [1, Lemma 4.1]), the absolutely summable sum

$$
\mathrm{e}^{-i \gamma h^{(\omega)}} \mathfrak{e}_{w}=\sum_{u \in \mathbb{Z}^{d}} \mathfrak{e}_{u}\left\langle\mathfrak{e}_{u}, \mathrm{e}^{-i \gamma h^{(\omega)}} \mathfrak{e}_{w}\right\rangle_{\mathfrak{h}}, \quad w \in \mathbb{Z}^{d}
$$

(see (42)-(43) ) and Lebesgue's dominated convergence theorem, in the limit $L \rightarrow \infty$ and for any $z \in \Lambda_{L / 2}$, there is an operator $\mathbf{R}_{\gamma, q, k}^{(L, \omega)} \in \mathcal{B}(\mathfrak{h})$ with vanishing operator norm as $L \rightarrow \infty$, uniformly with respect to $\omega \in \Omega, \lambda \in \mathbb{R}_{0}^{+}$and $\vartheta, \gamma$ in compact subsets of $\mathbb{R}_{0}^{+}$and $\mathbb{R}$, respectively, such that

$$
\mathbf{N}_{\gamma, q, k}^{(L, \omega)} \mathfrak{e}_{z}=\left(\mathbf{N}_{\gamma, q, k}^{(\infty, \omega)}+\mathbf{R}_{\gamma, q, k}^{(L, \omega)}\right) \mathfrak{e}_{z}
$$

where

$$
\begin{aligned}
\mathbf{N}_{\gamma, q, k}^{(\infty, \omega)} \mathfrak{e}_{z} \doteq & \sum_{x, y \in \mathbb{Z}^{d}}\left\{i\left\langle\mathfrak{e}_{x+e_{k}}, \Delta_{\omega, \vartheta} \mathfrak{e}_{x}\right\rangle_{\mathfrak{h}}\left\langle\mathfrak{e}_{y+e_{q}}, \Delta_{\omega, \vartheta} \mathfrak{e}_{y}\right\rangle_{\mathfrak{h}}\left\langle\mathfrak{e}_{x}, \mathrm{e}^{-i \gamma h^{(\omega)}} \mathfrak{e}_{y+e_{q}}\right\rangle_{\mathfrak{h}}\left\langle\mathfrak{e}_{y}, \mathrm{e}^{i \gamma h^{(\omega)}} \mathfrak{e}_{z}\right\rangle_{\mathfrak{h}} \mathfrak{e}_{x+e_{k}}\right. \\
& -i\left\langle\mathfrak{e}_{x+e_{k}}, \Delta_{\omega, \vartheta} \mathfrak{e}_{x}\right\rangle_{\mathfrak{h}}\left\langle\mathfrak{e}_{y}, \Delta_{\omega, \vartheta} \mathfrak{e}_{y+e_{q}}\right\rangle_{\mathfrak{h}}\left\langle\mathfrak{e}_{x}, \mathrm{e}^{-i \gamma h^{(\omega)}} \mathfrak{e}_{y}\right\rangle_{\mathfrak{h}}\left\langle\mathfrak{e}_{y+e_{q}}, \mathrm{e}^{i \gamma h^{(\omega)}} \mathfrak{e}_{z}\right\rangle_{\mathfrak{h}} \mathfrak{e}_{x+e_{k}} \\
& -i\left\langle\mathfrak{e}_{x}, \Delta_{\omega, \vartheta} \mathfrak{e}_{x+e_{k}}\right\rangle_{\mathfrak{h}}\left\langle\mathfrak{e}_{y+e_{q}}, \Delta_{\omega, \vartheta} \mathfrak{e}_{y}\right\rangle_{\mathfrak{h}}\left\langle\mathfrak{e}_{x+e_{k}}, \mathrm{e}^{-i \gamma h^{(\omega)}} \mathfrak{e}_{y+e_{q}}\right\rangle_{\mathfrak{h}}\left\langle\mathfrak{e}_{y}, \mathrm{e}^{i \gamma h^{(\omega)}} \mathfrak{e}_{z}\right\rangle_{\mathfrak{h}} \mathfrak{e}_{x} \\
& +i\left\langle\mathfrak{e}_{x}, \Delta_{\omega, \vartheta} \mathfrak{e}_{x+e_{k}}\right\rangle_{\mathfrak{h}}\left\langle\mathfrak{e}_{y}, \Delta_{\omega, \vartheta} \mathfrak{e}_{y+e_{q}}\right\rangle_{\mathfrak{h}}\left\langle\mathfrak{e}_{x+e_{k}}, \mathrm{e}^{-i \gamma h^{(\omega)}} \mathfrak{e}_{y}\right\rangle_{\mathfrak{h}}\left\langle\mathfrak{e}_{y+e_{q}}, \mathrm{e}^{i \gamma h} \mathfrak{e}_{z}\right\rangle_{\mathfrak{h}} \mathfrak{e}_{x} \\
& -i \delta_{x, z}\left\langle\mathfrak{e}_{y+e_{q}}, \Delta_{\omega, \vartheta} \mathfrak{e}_{y}\right\rangle_{\mathfrak{h}}\left\langle\mathfrak{e}_{x+e_{k}}, \Delta_{\omega, \vartheta} \mathfrak{e}_{x}\right\rangle_{\mathfrak{h}}\left\langle\mathfrak{e}_{y}, \mathrm{e}^{i \gamma h^{(\omega)}} \mathfrak{e}_{x+e_{k}}\right\rangle_{\mathfrak{h}} \mathrm{e}^{-i \gamma h^{(\omega)}} \mathfrak{e}_{y+e_{q}} \\
& +i \delta_{x+e_{k}, z}\left\langle\mathfrak{e}_{y+e_{q}}, \Delta_{\omega, \vartheta} \mathfrak{e}_{y}\right\rangle_{\mathfrak{h}}\left\langle\mathfrak{e}_{x}, \Delta_{\omega, \vartheta} \mathfrak{e}_{x+e_{k}}\right\rangle_{\mathfrak{h}}\left\langle\mathfrak{e}_{y}, \mathrm{e}^{i \gamma h^{(\omega)}} \mathfrak{e}_{x}\right\rangle_{\mathfrak{h}} \mathrm{e}^{-i \gamma h^{(\omega)}} \mathfrak{e}_{y+e_{q}} \\
& +i \delta_{x, z}\left\langle\mathfrak{e}_{y}, \Delta_{\omega, \vartheta} \mathfrak{e}_{y+e_{q}}\right\rangle_{\mathfrak{h}}\left\langle\mathfrak{e}_{x+e_{k}}, \Delta_{\omega, \vartheta} \mathfrak{e}_{x}\right\rangle_{\mathfrak{h}}\left\langle\mathfrak{e}_{y+e_{q}}, \mathrm{e}^{i \gamma h^{(\omega)}} \mathfrak{e}_{x+e_{k}}\right\rangle_{\mathfrak{h}} \mathrm{e}^{-i \gamma h^{(\omega)}} \mathfrak{e}_{y} \\
& \left.-i \delta_{x+e_{k}, z}\left\langle\mathfrak{e}_{y}, \Delta_{\omega, \vartheta} \mathfrak{e}_{y+e_{q}}\right\rangle_{\mathfrak{h}}\left\langle\mathfrak{e}_{x}, \Delta_{\omega, \vartheta} \mathfrak{e}_{x+e_{k}}\right\rangle_{\mathfrak{h}}\left\langle\mathfrak{e}_{y+e_{q}}, \mathrm{e}^{i \gamma h^{(\omega)}} \mathfrak{e}_{x}\right\rangle_{\mathfrak{h}} \mathrm{e}^{-i \gamma h^{(\omega)}} \mathfrak{e}_{y}\right\}
\end{aligned}
$$

It suffices now to use again (2) and (63) together with elementary manipulations in each sum of $\mathbf{N}_{\gamma, q, k}^{(\infty, \omega)}$ in order to arrive at the second assertion.

We are now in a position to show (57), at least for $|\gamma|, \vartheta \ll 1$, as a consequence of the next two lemmata:

Lemma 4.6 (Asymptotics for $\vartheta \ll 1$ )

For all $k, q \in\{1, \ldots, d\}, \vartheta, \lambda \in \mathbb{R}_{0}^{+}, \omega \in \Omega, \gamma \in \mathbb{R}$ and $z \in \mathbb{Z}^{d}$,

$$
\sum_{y \in \mathbb{Z}^{d}} \zeta_{z, y, z}=2 \Im \mathrm{m}\left\langle\left(s_{e_{k}}-s_{-e_{k}}\right) \mathfrak{e}_{z}, \mathrm{e}^{-i \gamma h^{(\omega)}}\left(s_{e_{q}}-s_{-e_{q}}\right) \mathrm{e}^{i \gamma h^{(\omega)}} \mathfrak{e}_{z}\right\rangle_{\mathfrak{h}}+\mathcal{O}(\vartheta), \quad \text { as } \vartheta \rightarrow 0,
$$

uniformly with respect to $\omega \in \Omega, \lambda \in \mathbb{R}_{0}^{+}$and $\gamma$ in compact subsets of $\mathbb{R}$. Note that $\vartheta$ is not necessarily 0 in definition of $h^{(\omega)}$.

Proof. By Lemma 4.5 at $\vartheta=0$, one directly computes that, for any $k, q \in\{1, \ldots, d\}, \lambda \in \mathbb{R}_{0}^{+}$, $\omega \in \Omega, \gamma \in \mathbb{R}, z \in \mathbb{Z}^{d}$ and $\vartheta=0$,

$$
\sum_{y \in \mathbb{Z}^{d}} \zeta_{z, y, z}=\sum_{y \in \mathbb{Z}^{d}} 2 \Im \mathrm{m}\left\langle\mathfrak{e}_{z+e_{k}}-\mathfrak{e}_{z-e_{k}}, \mathrm{e}^{-i \gamma h^{(\omega)}}\left(\mathfrak{e}_{y+e_{q}}-\mathfrak{e}_{y-e_{q}}\right)\right\rangle_{\mathfrak{h}}\left\langle\mathfrak{e}_{y}, \mathrm{e}^{i \gamma h^{(\omega)}} \mathfrak{e}_{z}\right\rangle_{\mathfrak{h}} .
$$

If $\vartheta \neq 0$ then one performs the same kind of computation in order to (trivially) deduce the assertion, by (33), Lemma 4.5 and $42-(43)$. 
Lemma 4.7 (Asymptotics for $|\gamma| \ll 1$ )

For all $k, q \in\{1, \ldots, d\}, \vartheta, \lambda \in \mathbb{R}_{0}^{+}, \omega \in \Omega, \gamma \in \mathbb{R}$ and $z \in \mathbb{Z}^{d}$,

$$
\begin{aligned}
2 \Im \mathrm{m}\left\langle\left(s_{e_{k}}-s_{-e_{k}}\right) \mathfrak{e}_{z}, \mathrm{e}^{-i \gamma h^{(\omega)}}\left(s_{e_{q}}\right.\right. & \left.-s_{-e_{q}}\right) \mathrm{e}^{\left.i \gamma h^{(\omega)} \mathfrak{e}_{z}\right\rangle_{\mathfrak{h}}} \\
& =2 \gamma \lambda \delta_{k, q}\left\{2 \omega_{1}(z)-\omega_{1}\left(z+e_{k}\right)-\omega_{1}\left(z-e_{k}\right)\right\}+\mathcal{O}\left(\gamma^{2}\right),
\end{aligned}
$$

as $|\gamma| \rightarrow 0$, uniformly with respect to $\omega \in \Omega$ and $\vartheta, \lambda$ in compact subsets of $\mathbb{R}_{0}^{+}$.

Proof. By (58), for any $\gamma \in \mathbb{R}$,

$$
\mathrm{e}^{i \gamma h^{(\omega)}}=\mathbf{1}_{\mathfrak{h}}+\sum_{n \in \mathbb{N}} \frac{\left(i \gamma h^{(\omega)}\right)^{n}}{n !}=\mathbf{1}_{\mathfrak{h}}+i \gamma h^{(\omega)}+\mathcal{O}\left(\gamma^{2}\right), \quad \text { as }|\gamma| \rightarrow 0,
$$

in the Banach space $\mathcal{B}(\mathfrak{h})$, uniformly with respect to $\omega \in \Omega$ and $\vartheta, \lambda$ in compact subsets of $\mathbb{R}_{0}^{+}$. The assertion then follows by direct computations using (2)-(3), (33) and the last equality.

Lemma 4.8 (Lower bounds on the Hilbert-Schmidt norm of $K_{\left\{\Lambda_{L}\right\},\left\{\mathbb{Z}^{d}\right\}}^{(\omega, \mathcal{E})}$ )

Take $\vartheta, \lambda, T \in \mathbb{R}_{0}^{+}, T \in \mathbb{R}^{+}, \mathcal{E} \in C_{0}^{0}\left(\mathbb{R} ; \mathbb{R}^{d}\right)$ with support in $[-T, 0]$ and $\vec{w} \stackrel{(}{=}\left(w_{1}, \ldots, w_{d}\right) \in \mathbb{R}^{d}$ with $\|\vec{w}\|_{\mathbb{R}^{d}}=1$. If $T$, $\vartheta$ are sufficiently small then

$$
\begin{aligned}
& \lim _{L \rightarrow \infty} \frac{1}{\left|\Lambda_{L}\right|} \mathbb{E}\left[\operatorname{Tr}_{\mathfrak{h}}\left(\left(K_{\left\{\Lambda_{L}\right\},\left\{\mathbb{Z}^{d}\right\}}^{(\cdot, \mathcal{E})}\right)^{*} K_{\left\{\Lambda_{L}\right\},\left\{\mathbb{Z}^{d}\right\}}^{(\cdot \mathcal{E})}\right)\right] \\
\geq & \frac{\lambda^{2}}{2} \operatorname{Var}\left[\int_{-\infty}^{0}\left\langle w^{(\cdot)}, \mathcal{E}(\alpha)\right\rangle_{\mathbb{R}^{d}} \alpha^{2} \mathrm{~d} \alpha\right]+\mathcal{O}\left(\vartheta^{2}\right)+\mathcal{O}\left(T^{4}\right),
\end{aligned}
$$

uniformly with respect to $\lambda$ in compact subsets of $\mathbb{R}_{0}^{+}$, where $w^{(\cdot)} \doteq\left(w_{1}^{(\cdot)}, \ldots, w_{d}^{(\cdot)}\right) \in \mathbb{R}^{d}$ is the random vector defined by

$$
w_{k}^{(\omega)} \doteq\left(2 \omega_{1}(0)-\omega_{1}\left(e_{k}\right)-\omega_{1}\left(-e_{k}\right)\right) w_{k}, \quad k \in\{1, \ldots, d\}, \omega \in \Omega .
$$

Proof. Fix all parameters of the lemma. Take any $L \geq 2$. Note that

$$
\operatorname{Tr}_{\mathfrak{h}}\left(\left(K_{\left\{\Lambda_{L}\right\},\left\{\mathbb{Z}^{d}\right\}}^{(\omega, \mathcal{E})}\right)^{*} K_{\left\{\Lambda_{L}\right\},\left\{\mathbb{Z}^{d}\right\}}^{(\omega, \mathcal{E})}\right) \geq \sum_{z \in \Lambda_{L / 2}}\left\|K_{\left\{\Lambda_{L}\right\},\left\{\mathbb{Z}^{d}\right\}}^{(\omega, \mathcal{E})} \mathfrak{e}_{z}\right\|_{\mathfrak{h}}^{2} \geq \sum_{z \in \Lambda_{L / 2}}\left|\left\langle\mathfrak{e}_{z}, K_{\left\{\Lambda_{L}\right\},\left\{\mathbb{Z}^{d}\right\}}^{(\omega, \mathcal{E})} \mathfrak{e}_{z}\right\rangle_{\mathfrak{h}}\right|^{2}
$$

By using (59)- 61) and Lemma 4.5, for any $z \in \Lambda_{L / 2}$, we have that

$$
\begin{aligned}
\left\langle\mathfrak{e}_{z}, K_{\left\{\Lambda_{L}\right\},\left\{\mathbb{Z}^{d}\right\}}^{(\cdot, \mathcal{e})} \mathfrak{e}_{z}\right\rangle_{\mathfrak{h}}= & \sum_{k, q=1}^{d} w_{k} \int_{-\infty}^{0}\{\mathcal{E}(\alpha)\}_{q} \int_{0}^{-\alpha} \sum_{y \in \mathbb{Z}^{d}} \zeta_{z, y, z} \mathrm{~d} \gamma \mathrm{d} \alpha \\
& +\sum_{k, q=1}^{d} w_{k} \int_{-\infty}^{0}\{\mathcal{E}(\alpha)\}_{q} \int_{0}^{-\alpha}\left\langle\mathfrak{e}_{z}, \mathbf{R}_{\gamma, q, k}^{(L, \omega)} \mathfrak{e}_{z}\right\rangle_{\mathfrak{h}} \mathrm{d} \gamma \mathrm{d} \alpha
\end{aligned}
$$

with $\mathbf{R}_{\gamma, q, k}^{(L, \omega)} \in \mathcal{B}(\mathfrak{h})$ satisfying 62 . Note that $\zeta_{z, y, z}$ is $\gamma$-dependent and its explicit expression is found in Lemma 4.5. If $T, \vartheta$ are sufficiently small then, by Lemmata 4.64.7, we deduce that, for any $z \in \Lambda_{L / 2}$,

$$
\begin{aligned}
\left\langle\mathfrak{e}_{z}, K_{\left\{\Lambda_{L}\right\},\left\{\mathbb{Z}^{d}\right\}}^{(\cdot, \mathcal{e})} \mathfrak{e}_{z}\right\rangle_{\mathfrak{h}}= & \lambda \sum_{k=1}^{d} w_{k} \int_{-\infty}^{0}\left\{2 \omega_{1}(z)-\omega_{1}\left(z+e_{k}\right)-\omega_{1}\left(z-e_{k}\right)\right\}\{\mathcal{E}(\alpha)\}_{k} \alpha^{2} \mathrm{~d} \alpha \\
& +\mathcal{O}(\vartheta)+\mathcal{O}\left(T^{2}\right)+\sum_{k, q=1}^{d} w_{k} \int_{-\infty}^{0}\{\mathcal{E}(\alpha)\}_{q} \int_{0}^{-\alpha}\left\langle\mathfrak{e}_{z}, \mathbf{R}_{\gamma, q, k}^{(L, \omega)} \mathfrak{e}_{z}\right\rangle_{\mathfrak{h}} \mathrm{d} \gamma \mathrm{d} \alpha
\end{aligned}
$$


uniformly with respect to $\omega \in \Omega$ and $\lambda$ in compact subsets of $\mathbb{R}_{0}^{+}$. By the translation invariance of the distribution $\mathfrak{a}_{\Omega}$ (see [1, Equations (1)-(2)]) and (62), it follows that

$$
\begin{aligned}
\lim _{L \rightarrow \infty} \mathbb{E}\left[\left|\left\langle\mathfrak{e}_{z}, K_{\left\{\Lambda_{L}\right\},\left\{\mathbb{Z}^{d}\right\}}^{(\cdot, \mathcal{E})} \mathfrak{e}_{z}\right\rangle_{\mathfrak{h}}\right|^{2}\right] & =\lambda^{2} \mathbb{E}\left[\left|\int_{-\infty}^{0}\left\langle w^{(\cdot)}, \mathcal{E}(\alpha)\right\rangle_{\mathbb{R}^{d}} \alpha^{2} \mathrm{~d} \alpha\right|^{2}\right]+\mathcal{O}\left(\vartheta^{2}\right)+\mathcal{O}\left(T^{4}\right) \\
& =\lambda^{2} \operatorname{Var}\left[\int_{-\infty}^{0}\left\langle w^{(\cdot)}, \mathcal{E}(\alpha)\right\rangle_{\mathbb{R}^{d}} \alpha^{2} \mathrm{~d} \alpha\right]+\mathcal{O}\left(\vartheta^{2}\right)+\mathcal{O}\left(T^{4}\right),
\end{aligned}
$$

uniformly with respect to $\lambda$ in compact subsets of $\mathbb{R}_{0}^{+}$. Thanks to 65, the assertion then follows. Note that

$$
\mathbb{E}\left[\int_{-\infty}^{0}\left\langle w^{(\cdot)}, \mathcal{E}(\alpha)\right\rangle_{\mathbb{R}^{d}} \alpha^{2} \mathrm{~d} \alpha^{2}\right]=0
$$

By combining Lemmata 4.4, 4.8 and 4.3, we directly obtain that, for any $\vartheta, \lambda, T \in \mathbb{R}_{0}^{+}$, $T, \beta \in \mathbb{R}^{+}, \mathcal{E} \in C_{0}^{0}\left(\mathbb{R} ; \mathbb{R}^{d}\right)$ with support in $[-T, 0]$ and $\vec{w} \in \mathbb{R}^{d}$ with $\|\vec{w}\|_{\mathbb{R}^{d}}=1$,

$$
\left.\partial_{s}^{2} \mathrm{~J}^{(s \mathcal{E})}\right|_{s=0} \geq \frac{1}{2\left(1+\mathrm{e}^{\beta(2 d(2+\vartheta)+\lambda)}\right)^{2}}\left(\lambda^{2} \operatorname{Var}\left[\int_{-\infty}^{0}\left\langle w^{(\cdot)}, \mathcal{E}(\alpha)\right\rangle_{\mathbb{R}^{d}} \alpha^{2} \mathrm{~d} \alpha\right]+\mathcal{O}\left(\vartheta^{2}\right)+\mathcal{O}\left(T^{4}\right)\right),
$$

provided that $T, \vartheta$ are sufficiently small. In particular, if

$$
\operatorname{Var}\left[\int_{-\infty}^{0}\left\langle w^{(\cdot)}, \mathcal{E}(\alpha)\right\rangle_{\mathbb{R}^{d}} \alpha^{2} \mathrm{~d} \alpha\right]>0
$$

then $\left.\partial_{s}^{2} \mathrm{~J}^{(s \mathcal{E})}\right|_{s=0}>0$. This last condition is easily satisfied: Because the variance of the sum (or the difference) of uncorrelated random variables is the sum of their variances, if the random variables $\omega_{1}(0), \omega_{1}\left(e_{1}\right), \omega_{1}\left(-e_{1}\right), \ldots, \omega_{1}\left(e_{d}\right), \omega_{1}\left(-e_{d}\right)$ are independently and identically distributed (i.i.d.), then

$$
\begin{aligned}
\mathbb{E}\left[\left|\int_{-\infty}^{0}\left\langle w^{(\omega)}, \mathcal{E}(\alpha)\right\rangle_{\mathbb{R}^{d}} \alpha^{2} \mathrm{~d} \alpha\right|^{2}\right]= & 2 \operatorname{Var}\left[(\cdot)_{1}(0)\right] \times\left(2\left(\int_{-\infty}^{0}\langle w, \mathcal{E}(\alpha)\rangle_{\mathbb{R}^{d}} \alpha^{2} \mathrm{~d} \alpha\right)^{2}\right. \\
& \left.+\sum_{k=1}^{d}\left(w_{k} \int_{-\infty}^{0}(\mathcal{E}(\alpha))_{k} \alpha^{2} \mathrm{~d} \alpha\right)^{2}\right)
\end{aligned}
$$

which is strictly positive as soon as $\mathcal{E} \neq 0$ and $\omega_{1}(0)$ is not almost surely constant, by Chebychev's inequality.

Acknowledgments: This work is supported by CNPq (308337/2017-4), as well as by the Basque Government through the grant IT641-13 and the BERC 2018-2021 program and by the Spanish Ministry of Science, Innovation and Universities: BCAM Severo Ochoa accreditation SEV-20170718, MTM2017-82160-C2-2-P. We thank S. Rodrigues for having pointed out [4].

\section{References}

[1] N.J.B. Aza, J.-B. Bru, W. de Siqueira Pedra and A. Ratsimanetrimanana, Accuracy of classical conductivity theory at atomic scales for free fermions in disordered media. J. Math. Pures Appl. 125 (2019) 209-246.

[2] D. K. Ferry, Ohm's Law in a Quantum World, Science 335(6064) (2012) 45-46. 
[3] B. Weber et al., Ohm's Law Survives to the Atomic Scale, Science 335(6064) (2012) 64-67.

[4] B. Zhang et al., Role of contacts in long-range protein conductance, PNAS 116(13) (2019) 5886-5891.

[5] J.-B. Bru, W. de Siqueira Pedra and C. Hertling, AC-Conductivity Measure from Heat Production of Free Fermions in Disordered Media. Archive for Rational Mechanics and Analysis 220(2) (2016) 445-504.

[6] J.-B. Bru and W. de Siqueira Pedra, From the 2nd Law of Thermodynamics to the ACConductivity Measure of Interacting Fermions in Disordered Media, M3AS: Mathematical Models and Methods in Applied Sciences 25(14) (2015) 2587-2632.

[7] J.-D. Deuschel and D. W. Stroock, Large Deviations, American Mathematical Soc., Providence, Rohde Island (1989).

[8] A. Dembo and O. Zeitouni, Large Deviations Techniques and Applications, 2nd ed. Springer-Verlag, New-York (1998).

[9] N. J. B. Aza, J.-B. Bru, W. de Siqueira Pedra, L. Müssnich, Large Deviations in Weakly Interacting Fermions I - Generating Functions as Gaussian Berezin Integrals and Bounds on Large Pfaffians. (2017) Preprint mp_arc 17-71.

[10] J.-B. Bru, W. de Siqueira Pedra and C. Hertling, Macroscopic Conductivity of Free Fermions in Disordered Media, Rev. Math. Phys. 26(5) (2014) 1450008-1-25.

[11] D. Goderis, A. Verbeure and P. Vets, Noncommutative central limits. Probab. Theory Related Fields 82 (1989) 527-544.

[12] D. Goderis, A. Verbeure and P. Vets, Theory of quantum fluctuations and the onsager relations, J. Stat. Phys. 56 (1989) 721-746.

[13] D. Goderis, A. Verbeure and P. Vets, About the mathematical theory of quantum fluctuations, In: Mathematical Methods in Statistical Mechanics. Leuven Notes Math. Theoret. Phys. Ser. A Math. Phys., 1, Leuven, Leuven Univ. Press, 1989, p. 31.

[14] D. Goderis, A. Verbeure and P. Vets, Quantum central limit and coarse graining. In: Quantum probability and applications, V, Vol. 1442 of Lecture Notes in Math. BerlinHeidelberg-New York, Springer, 1990, p. 178-193.

[15] D. Goderis, A. Verbeure and P. Vets, Dynamics of fluctuations for quantum lattice systems, Commun. Math. Phys. 128 (1990) 533-549.

[16] D. Goderis, A. Verbeure and P. Vets, About the exactness of the linear response theory, Commun. Math. Phys. 136 (1991) 265-283.

[17] André F. Verbeure, Many-Body Boson Systems, Springer-Verlag London Limited, 2011.

[18] J.-B. Bru and W. de Siqueira Pedra, Lieb-Robinson Bounds for Multi-Commutators and Applications to Response Theory, SpringerBriefs in Mathematical Physics, Vol. 13 (2017).

[19] W. Rudin, Functional Analysis. McGraw-Hill Science, 1991.

[20] H. Schulz-Baldes and J. Bellissard. A kinetic theory for quantum transport in aperiodic media. Journal of statistical physics 91(5-6) (1998) 991-1026. 
[21] J. Bellissard, A. van Elst, and H. Schulz-Baldes, The noncommutative geometry of the quantum Hall effect. J. Math. Phys. 35(10) (1994) 5373-5451.

[22] J.-M. Bouclet, F. Germinet, A. Klein and J.H. Schenker, Linear response theory for magnetic Schrödinger operators in disordered media, Journal of Functional Analysis 226 (2005) 301-372.

[23] A. Klein, O. Lenoble, P. Müller On Mott's formula for the AC-conductivity in the Anderson Model, Annal of Mathematics, 166 (2007) 549-577.

[24] A. Klein and P. Müller, The Conductivity Measure for the Anderson Model, Journal of Mathematical Physics, Analysis, Geometry 4 (2008) 128-150.

[25] A. Klein and P. Müller, AC-conductivity and Electromagnetic Energy Absorption for the Anderson Model in Linear Response Theory, Markov Processes Relat. Fields 21 (2015) 575-590.

[26] N. Dombrowski, F. Germinet, Linear response theory for random Schrodinger operators and noncommutative integration, Markov. Proc. Rel. F. 18 (2008) 403-426.

[27] E. Prodan, Quantum Transport in Disordered Systems Under Magnetic Fields: A Study Based on Operator Algebras. Applied Mathematics Research eXpress 2013(2) (2013) 176265.

[28] M. H. Brynildsen, H. D. Cornean, On the Verdet constant and Faraday rotation for graphene-like materials, Rev. Math. Phys. 25(4) (2013) 1350007-1-28.

[29] J.-B. Bru and W. de Siqueira Pedra, Microscopic Foundations of Ohm and Joule's Laws The Relevance of Thermodynamics. (2014). Mathematical Results in Quantum Mechanics, Proceedings of the QMath12 Conference, Eds.: P. Exner, W. König, H. Neidhardt, World Scientific (2014).

[30] J.-B. Bru, W. de Siqueira Pedra and C. Hertling, Heat Production of Non-Interacting Fermions Subjected to Electric Fields, Comm. Pure Appl. Math. 68(6) (2015) 964-1013.

[31] J.-B. Bru, W. de Siqueira Pedra and C. Hertling, Microscopic Conductivity of Lattice Fermions at Equilibrium - Part I: Non-Interacting Particles, J. Math. Phys. 56 (2015) 051901-1-51.

[32] J.-B. Bru and W. de Siqueira Pedra, Microscopic Conductivity of Lattice Fermions at Equilibrium - Part II: Interacting Particles, Letters in Mathematical Physics 106(1) (2016) 81-107.

[33] O. Bratteli and D.W. Robinson, Operator Algebras and Quantum Statistical Mechanics, Vol. II, 2nd ed., Springer-Verlag, New York (1996).

[34] C.-A. Pillet, "Quantum Dynamical Systems", Open Quantum Systems I: The Hamiltonian Approach, Lecture Notes in Mathematics Vol. 1880 edited by Attal S., Joye A., and Pillet C.-A. (Springer-Verlag, 2006).

[35] H. Araki, On Quasifree States of CAR and Bogoliubov Automorphisms, Publ. RIMS, Kyoto Univ., 6 (1970/71), 385-442.

[36] A. Ratsimanetrimanana, Equilibrium and Transport Properties of Quantum Many-Body Systems, PhD Thesis, Universidad del País Vasco, 2019. 
[37] J.-B. Bru and W. de Siqueira Pedra, Universal Bounds for Large Determinants from Non Commutative Hölder Inequalities in Fermionic Constructive Quantum Field Theory, Mathematical Models and Methods in Applied Sciences 27(10) (2017) 1963-1992. 